\title{
Invariant and Consistent Redundancy by Large Admissible Perturbations
}

\author{
Byungsik Kang, Eleni Beyko \& Michael M. Bernitsas
}

Department of Naval Architecture and Marine Engineering. The University of Michigan, North Campus, Ann Arbor, Michigan 48109-2145, USA

(Received 17 March 1991; revised version received 2 May 1991; accepted 20 August 1991)

\begin{abstract}
Structural perturbation theory has been developed over the past 16 years to relate two structural states modeled by the same Finite Element (FE) model but described by different values of the design variables. Relating an intact/ damaged (initial) structure to a limit state structure produces the reserve/ residual redundancy. Invariant and consistent redundancy, redundancy functions, and injective mappings are defined and related to the design variables. General perturbation equations are derived to relate the two states and produce failure surface equations. Individual and joint failure points are identified and redundancy is computed without linearization of failure surfaces, enumeration of failure paths, trial and error, or repeated $F E$ Analyses (FEAs). This is achieved by large admissible perturbations using a prediction-correction algorithm and postprocessing FEA results of the initial structure only. The latter may differ from the limit state structure in stiffness, mass, geometry, or response by as much as 100-300\% depending on the size of the FE model. Structural perturbation theory treats discrete and continuous structures as the FE method does; modeling of the structure as a simplified system of components is not needed. To introduce this new approach to redundancy, modal dynamic and static deflection failure criteria are used in the elastic range. Numerical applications on a beam, a small, and a large offshore tower are used to test the method. Future developments and impact to design are discussed as the new methodology introduces an alternative to systems reliability and stochastic $F E$.
\end{abstract}

Marine Structures 0951-8339/92/\$05.00 @ 1992 Elsevier Science Publishers Ltd, England. Printed in Great Britain. 
Key words: redundancy in performance, stiffness, mass, geometry, invariant and consistent redundancy injections, failure equations, failure point identification, large continuous structures, large admissible perturbations, FEA.

\section{NOTATION}

$c_{i j}$

$d, e$

$\operatorname{dof}(\mathbf{s})$

E

FE

FEA

$g, l, m$

$[k],\left[-K_{-}\right]$

$\left[k_{e}\right]$

$\boldsymbol{K}_{\boldsymbol{i}}$

l

LEAP

$\mathscr{H}$

$[m],[-M]$

$\left[m_{e}\right]$

$M_{\mathrm{i}}$

$n$

$N$

$n_{\mathrm{a}}$

$n_{\mathrm{u}}, n_{\omega}, n_{\phi}$

$n_{\mathrm{r}}$

$n_{\sigma}, n_{\mathrm{b}}, n_{\phi \mathrm{b}}$

$p$

PAR

RESTRUCT

S1, S2 admixture coefficient for participation of the $j$ th mode to changes in the $i$ th mode index for design and model variables, respectively degree(s) of freedom Young's modulus

Finite Element

Finite Element Analysis

indices for geometry, load, and material variables, respectively

global and generalized stiffness matrices

stiffness matrix of element or group of elements related to property $e$

$i$ th component of $\left[-K_{-}\right]$

index denoting quantity in increment $l, l=1,2, \ldots, N$

Large admissible perturbation

FE model used in redundancy analysis

global and generalized mass matrices

mass matrix of element or group of elements related

to property $e$

$i$ th component of [-M-]

number of degrees of freedom in FE model

number of increments in incremental algorithm

number and admissibility constraints

number of displacement, frequency, and dynamic

model node failure criteria

number of extracted structural modes used in

RESTRUCT

number of stress, buckling loads, and buckling modal

node failure criteria

number of model variables

Perturbation Approach to redundancy

program for Redesign of STRUCTures

initial and other structural states, respectively,

modeled by $\mathscr{M}$ 


$\begin{array}{ll}\mathrm{S} 2_{i}, \mathrm{~S} 2_{i}^{*} & \text { limit state and failure point, respectively, on limit state } i \\ \{u\} & \text { nodal static displacement vector } \\ a_{e} & \text { fractional change to element or group property } e \\ \alpha_{e}^{+}, \alpha_{e}- & \text { upper and lower bounds for } a_{e} \\ \delta & \text { prefix denoting incremental change } \\ \Delta & \text { prefix denoting total change } \\ \{\sigma\} & \text { equivalent stress nodal vector } \\ {[\phi]} & \text { matrix of mode shape vectors } \\ \phi_{k i} & k \text { th degree of freedom } i \text { th mode shape } \\ \{\psi\}_{i},\left\{\psi_{b}\right\}_{i} & \text { ith dynamic and buckling mode, respectively } \\ \omega_{i} & i \text { th natural frequency } \\ \Gamma_{-}- & \text {indicates diagonal matrix } \\ ()^{\prime} & \text { prime denotes structural property of state S2 }\end{array}$

\section{INTRODUCTION AND BACKGROUND}

To put into perspective the redundancy concept and the computational methodology introduced in this paper, the state of the art in definition and computation of redundancy is reviewed in Section 1.1. Structural perturbation theory is reviewed in Section 1.2 with emphasis on the principle underlying large admissible perturbations, their potential to solve the problems of failure point identification and redundancy, and what problems have been solved up to date using that theory. The large admissible perturbation approach to redundancy is introduced in Section 2 by defining its goals and describing its present capabilities and limitations and its potential for further development. Several definitions are provided in Section 2.3 leading to the concepts of invariant and consistent redundancy, and redundancy injections, mappings and measures. In Section 3, redundancy is defined with respect to global and element stiffness and mass, geometry, performance, and strength. Oneto-one relations between all those redundancy definitions are established in the structural perturbation theory by preserving element connectivity in the finite element model and ensuring that all large perturbations are admissible producing a real structure. In Section 4, modal dynamic and static deflection failure criteria are introduced and quantified and the corresponding global failure equations are derived by developing general perturbation equations. Stress and global buckling failure criteria which are under development are presented. The solution algorithm developed to identify the failure point for each failure criterion is outlined in Section 5. Large admissible perturbations are used to identify failure points without trial and error or path enumeration and by 
postprocessing results of a single finite element analysis, that of the initial structure. Numerical applications presented in Section 6 show that the initial and limit state structures may be far apart (100-300\%) in stiffness, mass, performance or geometry. Three structures are used in the numerical applications: a 10-element 48-dof clamped-hinged beam, a 104-element 192-dof offshore tower and a 605-element 810-dof offshore tower. Finally, our current research efforts and the impact of the developed methodology to design are discussed.

\subsection{Redundancy}

In the literature on marine structural reliability, most papers study redundancy in the sense of residual strength with respect to collapse ${ }^{1-7}$. Historically, however, redundancy has been defined in a variety of other ways. (R1) External redundancy is defined as the number of excess reaction components needed for structural stability ${ }^{2}$. (R2) Internal redundancy for discrete structures is closely related to determinancy ${ }^{2}$. (R3) Reserve redundancy is defined as the margin between the design load and the limit state ${ }^{3}$. (R4) Global redundancy refers to failure of the overall structure 4 . (R5) Local redundancy is synonymous with reserve strength of individual structural members and joints ${ }^{4}$. (R6) Serviceability redundancy addresses the issue of reduction of the operational threshold rather than collapse?.

Focusing on redundancy in the sense of reserve or residual strength with respect to collapse, we find several measures for quantifying redundancy. Deterministic measures are as follows. (M1) The redundant factor defined as the ratio of the intact structure strength over the difference of damaged from intact strength ${ }^{1}$. (M2) The reserve resistance factor defined as the environmental load exerted on the undamaged structure at collapse over the design environmental load'. (M3) The residual resistance factor, $\mathrm{RIF}=\mathbf{R}_{\mathrm{ult} \text { d }} / \mathbf{R}_{\mathrm{ult}}$ where $\mathbf{R}_{\text {ult.d, }}, \mathbf{R}_{\mathrm{ult}}$ are the ultimate strengths of the damaged and intact structures, respectively ${ }^{2}$. (M4) The deterministic system factor, DSF $=\lambda_{\max } / \lambda_{1}$, where $\lambda_{\max }$ and $\lambda_{1}$ are the deterministic first failure capacity and system capacity, respectively ${ }^{8}$. Probabilistic measures are as follows. (M5) The complexity, the redundancy, and the net system factors ${ }^{9}$. (M6) The conditional probability of failure given that one structural member has failed ${ }^{10}$. (M7) The conditional probability of failure given that one or more members have failed simultaneously ${ }^{11.12}$. (M8) The residual system reliability defined as the minimum of the reliabilities of the system when any single member is damaged ${ }^{13}$. (M9) Robustness, defined as the ratio of the 
conditional probability of failure of the structure with any one member damaged over the probability of failure of the intact structure ${ }^{10}$.

Even if we focus on reserve and residual redundancy with respect to collapse only, the existing measures will depend on the following factors: (F1) the type of structure ${ }^{2}$; (F2) whether the structure is discrete or continuous $^{2} ;(F 3)$ the type of loads applied ${ }^{2} ;(F 4)$ the idealization of the structure; (F5) the strength modeling of members (ductile or brittle) ${ }^{14}$; and the strength of individual members (balanced or unbalanced) ${ }^{8}$.

Presently, research in structural redundancy analysis faces the following challenges. (C1) Introduce a rational definition of redundancy from the structural point of view so that a redundancy measure can be related directly to geometric and material properties of elements ${ }^{1.2 .15-19}$. This would allow implementation of global redundancy design criteria at the early stages of element design. To explain this concept, consider the two traditional approaches to structural reliability design, that is the element and the system approaches ${ }^{3}$. In the former, selection of individual member reliability is empirical. In the systems approach, the overall system reliability is specified and an optimization with a trial and error technique is required to achieve that goal. A rational definition of redundancy should reveal the relation between the overall system reliability and element properties. In such a case, redesign of elements to achieve the target reliability of a structure will be possible without simplification of the structure or trial and error. (C2) Define an invariant redundancy measure that will not depend on factors F1-F6 listed in the previous paragraph ${ }^{1.2}$. (C3) Use sophisticated structural analysis in redundancy computations. This implies that finite element analysis (which is a standard technique in structural analysis and design) must be used. The alternative, that is modeling of a structure as a simplified system of structural components is in general not accurate, particularly for continuous structures ${ }^{8-11}$. (C4) Quantify global failure and derive global failure equations as functions of the design variables. Global refers to a property of the overall structure like natural frequency, normal mode, static deflection, static stress, buckling, collapse. Lack of global failure equations is a major obstacle to structural redundancy and reliability analysis ${ }^{20.21}$. (C5) Develop computationally efficient procedures for redundancy analysis of large scale structures. That is, enumeration of paths to failure and repeated FEAs must be avoided.

\subsection{Structural perturbation methods}

The methodology introduced in this paper, for redundancy analysis of large scale discrete or continuous structures is based on the large 
admissible perturbation theory. This theory has evolved in the past 7 years from the linear perturbation techniques introduced in 1975 for the purpose of redesigning a structure with undesirable modal dynamics characteristics ${ }^{22}$. Redesign is the process of defining an objective structure of given specifications starting from a baseline structural design which must be improved. Optimization or minimum error solution techniques may be used to define the objective structure depending on whether the problem is underdetermined or overdetermined, respectively. Redesign by perturbation differs from classical structural optimization in problem formulation, in expressing design goals in terms of design variables, in solving the problem without repeated $\mathrm{FE}$ analyses, in achieving large structural changes, and in meeting many different types of design objectives (frequency, modes, deflections, stresses, buckling, etc. $)^{23-26}$.

Linear perturbation methods allow for small differences between baseline and objective design in modal dynamic and structural properties. Redesign is achieved by perturbation without trial and error and by postprocessing only FEA results of the baseline design ${ }^{22-24.27 .28}$. Related to perturbation are design sensitivity methods ${ }^{29}$. They allow for small structural changes and in that respect they are equivalent to linear perturbation methods. Nonlinear perturbation methods were then developed to allow for large changes in modal dynamic response and structural element sizing ${ }^{25.26 .30-33}$. By the definition of the redesign process, it is clear that perturbation methods relate two structural states, the baseline and objective structures ${ }^{26}$. Other very important two-state problems in structural analysis and design are FE model correlation ${ }^{33 .}{ }^{34}$, structural system identification ${ }^{28}$, non-destructive testing ${ }^{28}$, failure mode identification, redundancy, and reliability. In the last three problems, the two states involved a re the intact or damaged structure and a limit state. In perturbation theory, relations between two structural states are derived, failure criteria are quantified, and algorithms are developed to define one of the two structural states in terms of the other.

Research in perturbation theory presently has three major goals. The first is to improve the solution algorithm so that it can be applied to larger scale structures and for larger differences between the two structural states in structural properties and response particulars. For that purpose incremental prediction-correction algorithms have been developed ${ }^{25.30 .31}$, which have been improved by the introduction of admissibility constraints $^{26}$ and the definition of cognate admissible space ${ }^{33}$. Those advancements have made it possible for large admissible perturbation algorithms to relate two states which may differ by $100-300 \%$ in properties and response. Identification of the objective state is achieved without trial and error and with only one FE analysis, that of the baseline 
structure. A major advantage of the large admissible perturbation theory is that it relates response changes to structural element sizes. Thus, the stiffness and mass matrices produced correspond to a real structure. Presently, substructuring and supercomputing are being implemented to make the large admissible perturbation algorithm faster and applicable to larger scale structures.

The second research goal is to derive equations for new relational criteria. Natural frequency criteria were first introduced followed by dynamic mode shape criteria ${ }^{22-24.27 .28 .30}$. Equations for static deflection criteria were then derived and algorithms implementing all of the above were developed ${ }^{25.26 .33 .34}$. Global buckling and stress relations have been developed recently (see Section 4.3).

The third research goal is to solve new two-state problems. The nondestructive testing problem was addressed by linear perturbation methods ${ }^{28}$. Centrifugal and Coriolis effects were introduced by perturbation in optimization of turbine blades ${ }^{35}$. Significant progress was made towards the solution of the problems of redesign and the FE model correlation which have been studied extensively in aerospace engineering $^{26.33}$. The FE model correlation problem was also solved for offshore structures where the external hydrodynamic force is a function of the design variables ${ }^{34}$. This paper represents a first step towards development of a large admissible perturbation methodology for solution of the failure point identification and redundancy problems.

\section{LARGE ADMISSIBLE PERTURBATION APPROACH TO REDUNDANCY}

The previous review of structural redundancy and structural perturbation theory was intended to provide the reader with insight into the potential of large admissible perturbation theory to address the current challenges in redundancy analysis of large scale continuous structures. In this chapter, the principle of LargE Admissible Perturbation (LEAP) theory is presented in Section 2.1; the major points of the Perturbation Approach to Redundancy (PAR) a re stated in Section 2.2 and presented in Sections 3-5; several definitions are provided in Section 2.3 to set the ground for the PAR methodology introduced in this paper.

\subsection{The LEAP principle}

As explained in Section 1.2, the purpose of LEAP is to relate two structural states, an initial state (S1) and an objective state (S2). S1 is analyzed by a FE method as shown in Fig. 1. S2 is characterized by 


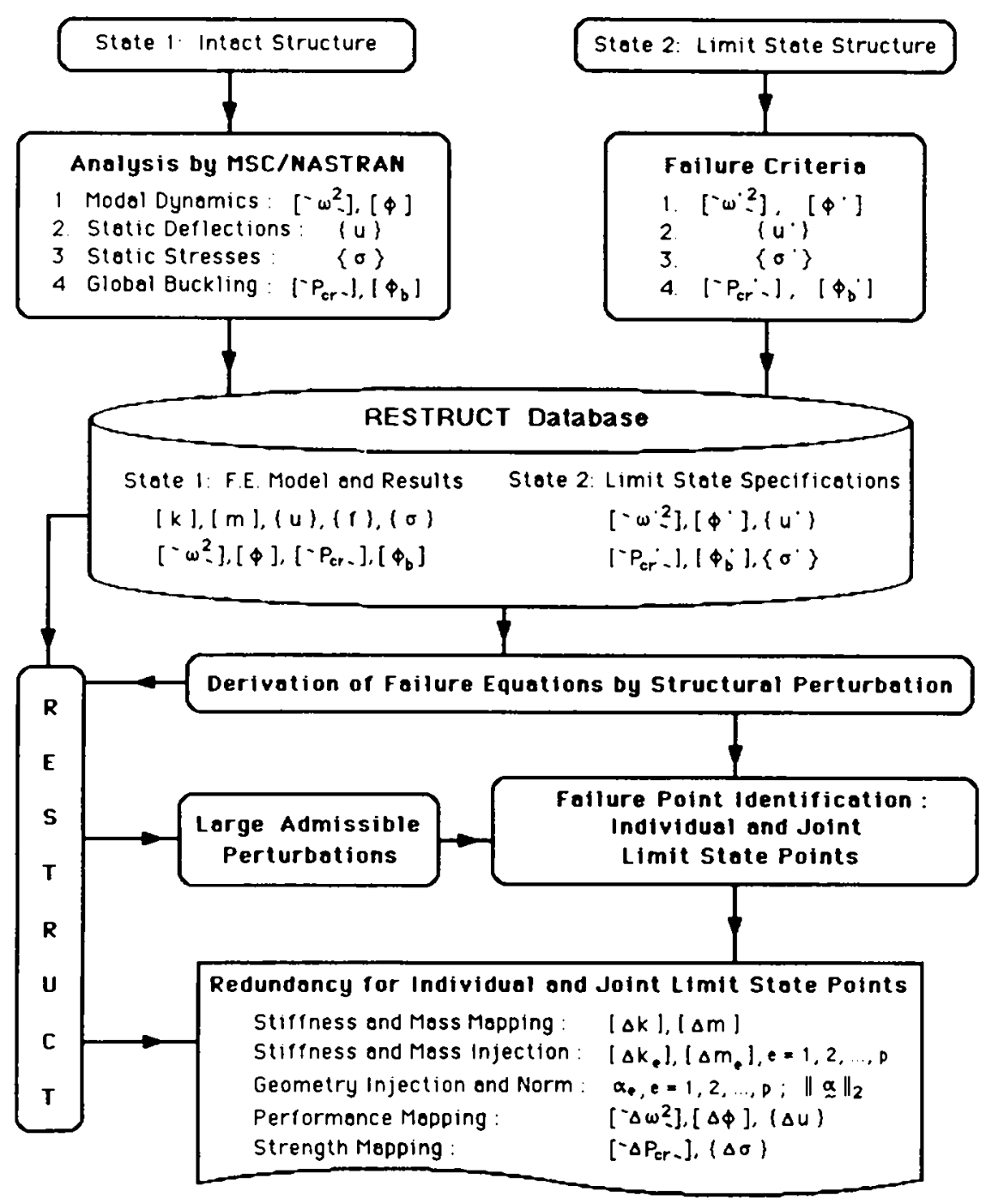

Fig. 1. Failure point identification and redundancy by perturbation.

performance specifications. LEAP methods identify $\mathrm{S} 2$ by postprocessing FE model data on S1 using S2 specifications. It should be emphasized again that $\mathrm{S} 2$ is defined by its desired performance not by values of design variables; that is, LEAP theory deals with two-state redesign not reanalysis problems. The latter were studied intensely in the early 1960 s to avoid running FE codes every time a small change in the stiffness and/ or mass matrix of a model was made. Faster computers and development of preprocessors, have made reanalysis unattractive. In fact, reanalysis 
problems can be solved by LEAP theory since it develops general perturbation equations relating S2 to S1 (see Section 4). In that case, the reanalysis LEAP algorithm would be much simpler than the redesign LEAP algorithm presented in Section 5, because design variables rather than desired structural performance would be specified.

To understand if a specific two-state problem in analysis or design (like redesign, FE model correlation, redundancy, etc.) can be addressed by LEAP theory, the following principle should be inferred: 'General perturbation equations can be developed to relate two structures $-\mathrm{S} 1$ and $\mathrm{S} 2$ - which are modeled by the same FE model but represented by different values of the design variables. Then, an incremental predictioncorrection algorithm can be developed to define structure S2 by postprocessing data of the FE analysis of structure S1.'

It is obvious that LEAP theory can solve a wide variety of problems in analysis and design that are presently solved by trial and error and repeated FE analyses. The redundancy problem is a two-state problem where $\mathbf{S} 1$ is the initial (intact/da maged) structure and $\mathbf{S} 2$ is a failure point on a limit surface. LEAP theory can address this problem as explained in the next section.

\subsection{The PAR concept}

The purpose of the methodology introduced in this paper is to meet challenges C1-C5 defined in Section 1.1. Its major points and their relation to those challenges are stated below and explained in Sections 3-5.

First, a structure is modeled and analyzed by a FE method (see Fig. 1). Large scale discrete or continuous structures are handled by general purpose FE codes, like MSC/NASTRAN.V64 used in this work. Then, redundancy is defined in a way consistent with structural design needs and invariant with respect to factors F1-F6 listed in Section 1.1. This procedure addresses challenges $\mathrm{C} 1-\mathrm{C} 3$ as explained in Section 3. Second, specify a failure criterion, quantify it, and derive global failure equations which represent the corresponding limit state surface. The latter is achieved by the general perturbation equations which relate state S2 to S1. Thus, challenge C4 is treated as shown in Section 4. Third, develop a large admissible perturbation algorithm to identify state S2*, the nearest failure point to $S 1$, by postprocessing data of only one FEA, that of state S1. This algorithm is outlined in Section 5 and addresses challenge $\mathrm{C} 5$.

By meeting challenge C3, the Perturbation Approach to Redundancy (PAR) brings the level of sophistication of structural analysis in 
redundancy computations up to par with standard analysis and design practice.

\subsection{Definitions}

After reviewing the literature pertinent to structural redundancy, it is considered necessary to establish the terminology used in this work by repeating some definitions and introducing a few new ones. This task is undertaken below. Classification of terms also helps to evaluate the introduced methodology, its potential and limitations, and its contributions to design beyond those of the two major methods of structural reliability, namely the systems approach ${ }^{9-11}$ and the stochastic FE method $^{36.37}$.

\section{Structural model and redesign}

The structure under consideration, whether small or large, discrete or continuous, and regardless of its stress-strain model, and external load is modeled and analyzed by the FE method.

(D1) Structural model. " is defined as the finite element model of the initial structure for which finite element a nalyses have been performed. That is, / refers to the numerical model not the physical structure or the mathematical model.

(D2) State $S 1$ is that of the initial (intact or damaged) structure for which model. " has been developed and run by a general purpose FE code. In LEAP-PAR theory, S1 represents the only state which has been analyzed by a FE code.

(D3) Structural analyses of . I" are those performed by the FE code for State S1. Such analyses may be modal dynamic, static, global buckling etc. What type of analyses are needed, depends on the failure criteria used in redundancy analysis.

(D4) State $S 2$ is any state which can be modeled by . " except S1. That is, S2 represents any structure which can be modeled by ." but represented by different values of the model variables. The question that arises here, is how flexible . / is, whether elements can be damaged, deleted or added, and if element strength can be modified without having to redefine state $\mathrm{S} 1$. To discuss these issues the following definitions are provided.

(D5) Redesign is the process of finding a structure S2 to satisfy certain design objectives, starting from state S1. LEAP theory does so without trial and error without any additional FEAs ${ }^{26}$.

(D6) Resizing is redesign where only structural element properties are allowed to change. That is, the grid of . " does not change. Nodes and 
degrees of freedom remain the same; structural elements are not eliminated or added.

(D7) Reshaping is redesign where, in addition to resizing, structural elements may be deleted or added, and element stress-strain models may be changed within the constraints of the grid of . II. The process of reshaping is needed when structural elements must be added to improve a structural design, or reach plasticity (ductile elements), or eliminated due to fracture (brittle elements).

(D8) Topological redesign is the process where, in addition to reconfiguration, the grid of ." is allowed to change.

Resizing is presently implemented in code RESTRUCT (Redesign of STRUCTures) and in the numerical applications in this paper. Reshaping and topological redesign are within the capability of LEAP theory but have not been implemented in RESTRUCT as yet ${ }^{38}$. Reshaping is needed after failure detection (element fracture or plasticity) for model accommodation/reconfiguration which are necessary in progressive element failure until global structural failure. Reshaping can be implemented readily in the general perturbation equations (see Section 4) and in code RESTRUCT, is presently under development. It should be noted that in our methodology, element failure does not dictate when and if state $\mathrm{S} 1$ must be redefined, that is $/ /$ reshaped and run again. Such an event depends on loss of accuracy of the modal expansion base (see Section 4.1) which may occur if perturbations become too big; like $100-300 \%$ changes.

The next phase in development of . $/$ will require topological redesign. The current version of RESTRUCT. V3 ${ }^{38}$, cannot perform topological redesign because it postprocesses FE results of MSC/NASTRAN.V64 which is the resident NASTRAN version in the University of Michigan secondary (system UB) mainframe computer (IBM 3090). We have developed, however, RESTRUCT.V4 running on the San Diego supercomputer center and postprocessing data of MSC/NASTRAN.V66 which has superlement capability. This provides the required flexibility for topological redesign in LEAP theory.

(D9) Substructure is a connected part of the structure. A substructure is delimited by its boundary; at least part of that boundary is an interface with other substructures. For example the deck of an offshore structure is a substructure and its lower boundary interfaces with the supporting tower.

(D10) A structural group is a set of structural elements. A group may not be connected and therefore may not be a substructure. A substructure may be treated as a group. The elements of a group have some common characteristic which defines the group. For example, in a redesign 
process of an offshore tower (see Figs. 6, 7 and Tables 3, 5) the tower legs may be grouped together so that they remain identical after redesign. For manufacturability purposes, identical braces may be grouped together in order to remain identical after redesign.

(D11) A structural element is a finite element in . "I. That is, a structural member, beam, plate, etc., may be modeled in . " by one or more finite elements.

Variables and vector spaces

Several quantities may be variable in . $/$; they are defined and classified below.

(D12) Geometric variables $a_{g}, g=1,2, \ldots, \mathrm{G}$; are those required to define uniquely the geometry of . "I. Two issues are important here. First, in resizing and reshaping, geometric variables describe cross-sections of elements since the grid." is fixed. Second, those variables are continuous and allow for continuous variation of element geometry; there is no need to limit element performance to a binary state, that is operational or failed.

(D13) Load variables $a_{l}, l=1,2, \ldots L$; are the variables in . " that uniquely define the load exerted on the structure. Presently, time independent load is implemented in RESTRUCT ${ }^{38}$ in static loading and buckling.

(D14) Material variables $a_{m}, m=1,2, \ldots, M$; are those required to define the stress-strain model of individual elements or substructures and their mass. Density $\rho$, Young's modulus $\mathrm{E}$, and $\sigma_{\mathrm{y}}$ are examples of material variables.

(D15) Design variables $a_{d}, d=1,2, \ldots, D$; are all variables needed to define uniquely any structure (S1 or S2) modeled by. II. That is, the set of design variables is the union of the geometry and material variable sets.

(D16) Model variables $a_{e}, e=1,2, \ldots, p$; are all variables required to define. II. The set of model variables is the union of the sets of design and load variables. All variables must be independent. For example the weight of a structure which contributes to static loading and buckling can be expressed in terms of geometry and material variables and consequently is not an $a_{e}$ variable.

(D17) Model space is the p-dimensional vector space defined by the model variables, $a \in h^{p}$. Similarly, we may define the geometry, load, material, and design spaces.

\section{Failure}

Terms pertinent to structural failure are defined in this subsection.

(D18) The failure criterion characterizes the way in which the 
structure fails to perform a particular task. Buckling, yielding, collapse, large deflections, low natural frequencies are failure criteria. They also define the structural analyses to be performed (see D3).

(D19) Failure or limit hypersurface corresponding to a failure criterion is the set of points, in the space of model variables, where failure may occur according to that criterion. In three dimensions, the limit hypersurface becomes a limit surface.

(D20) Quantificiation of a failure criterion is the assignment of a specific value to a failure criterion. In failure analysis, quantification may not always be necessary; e.g. 'global Euler buckling' is a sufficient description of a failure criterion. FE analysis will specify the first eigenvalue for buckling. In redundancy design, on the other hand, quantification may be necessary. For example, in the problem 'Design a structure to have a specified redundancy in global Euler buckling for a given compressive load $P_{\mathrm{cr}}, P_{\mathrm{cr}}$ quantifies the failure criterion, and it is required.

(D21) Failure or limit hypersurface equation $g_{i}(\mathbf{a})=0, a \in \mathscr{p}$ is the equation of the failure or limit hypersurface defined in the model vector space by failure criterion $(i)$ and its quantification, whenever the latter is necessary. In LEAP-PAR theory such equations are the general perturbation equations (see Section 4).

(D22) Failure point $\mathrm{S} 2_{i}^{*}$ is a point on the limit hypersurface $(i)$ where failure actually occurs; more than one such point may exist for given S1 and $g_{i}(\mathbf{a})=0$. The term failure mode used in the systems approach to indicate the sequence of element failures to structural collapse has no meaning in PAR. In PAR, the structure continuously depends on $a_{e}$ and any sequence of element failure does not define $S 2_{i}^{*}$ unless it is accompanied by information regarding the deteriorated or random state (geometry and material) of all structural elements. That is, the set of failure modes is not a denumerable set; it is indeed, as in a real structure, a multi-dimensional continuum. This continuum need not to be defined in PAR because failure hypersurfac equation $g_{i}(a)=0$ can be derived by perturbation and $S 2_{i}^{*}$ can be computed by a LEAP algorithm.

(D23) Survivability failure: this type of failure results in loss of the structure. Partial or total collapse of the structures falls in this category?

(D24) Serviceability failure: this type of failure results in service interruption ${ }^{7}$. Natural frequencies in the range of wave excitation, undesirable dyna mic modes, large deflections, or elastic buckling refer to ways in which a structure may lose its ability to perform its intended service.

(D25) Global failure refers to loss of the ability of a structure as a whole to perform a function ${ }^{4}$. Such failure is always associated with a global 
structural performance. Global Euler buckling, reduction of a natural frequency, collapse, excessive static deflection or stress, are examples of global failure.

(D26) Local failure refers to individual structural elements. Local failure is considered in LEAP theory in the process of failure detection and model reconfiguration. In that sense it does not constitute a major step in the computation of failure point $S 2_{i}^{*}$.

Regarding global and local failure, it should be stressed that any local event, like randomness in a member (corrosion, deterioration, etc.) affects the entire model and stress redistribution, and is taken into account inherently in the perturbation equations relating S2 to S1. Further, any deterioration is a continuous process until element fracture; it is not a binary state.

\section{Redundancy}

Our purpose is to provide consistent and invariant definitions of redundancy that can be used in design. This task is performed in Section 3. Here, we define the mathematical tools ${ }^{39}$ needed for defining redundancy. More specifically, the terms redundancy mapping, redundancy injection, redundancy norm, redundancy, consistent, and invariant are defined with the help of Fig. 2.

Let $X \subset . \mathscr{P}^{\mathrm{P}}$ be the model space (or space of the . I/ variables) and $A \subset X$ be the space of all real structures modeled by . $\mathbb{A} . A$ is a subset of $X$ because not all values of model variables represent a real structure; e.g. a geometric variable $a_{g}$ cannot be negative; when $a_{g}=0$ the corresponding element is removed from the structure. Structural states $\mathrm{S} 1$ and $\mathrm{S} 2$ are defined in $A$. The limit surface $L_{i}$, is a subject of $A$, that is $L_{i} \subset A$.

(D27) A mapping $T^{\delta}$, called difference mapping, is defined from $A$ into $Y \subset \mathscr{R P}$ so that

$$
\begin{aligned}
& T^{\delta}: A=\mathcal{H}\left(T^{\delta}\right) \rightarrow Y \subset \mathscr{p}^{\mathrm{p}} \\
& \mathbf{a} \mid \rightarrow T^{\delta} \mathbf{a}=a
\end{aligned}
$$

and

$$
a=\mathbf{a}_{2}-\mathbf{a}_{1}
$$

where $\mathcal{J}\left(T^{\delta}\right)$ is the domain of the transformation, and $\mathbf{a}_{1}$ and $\mathbf{a}_{2}$ define structural state $S 1$ and $S 2$, respectively.

The range of $T^{\delta}, \mathscr{R}\left(T^{\delta}\right)$ is the set of all images of $A$; that is

$$
B=\mathscr{h}\left(T^{\delta}\right)=\left\{\mathbf{y} \in Y \mid \mathbf{y}=T^{\delta} \mathbf{x} \quad \text { for some } \mathbf{x} \in \mathcal{S}\left(T^{\delta}\right)\right\}
$$

This transformation is an injection (injective mapping or one-to-one) and therefore no information is lost in the process. A structural state may 


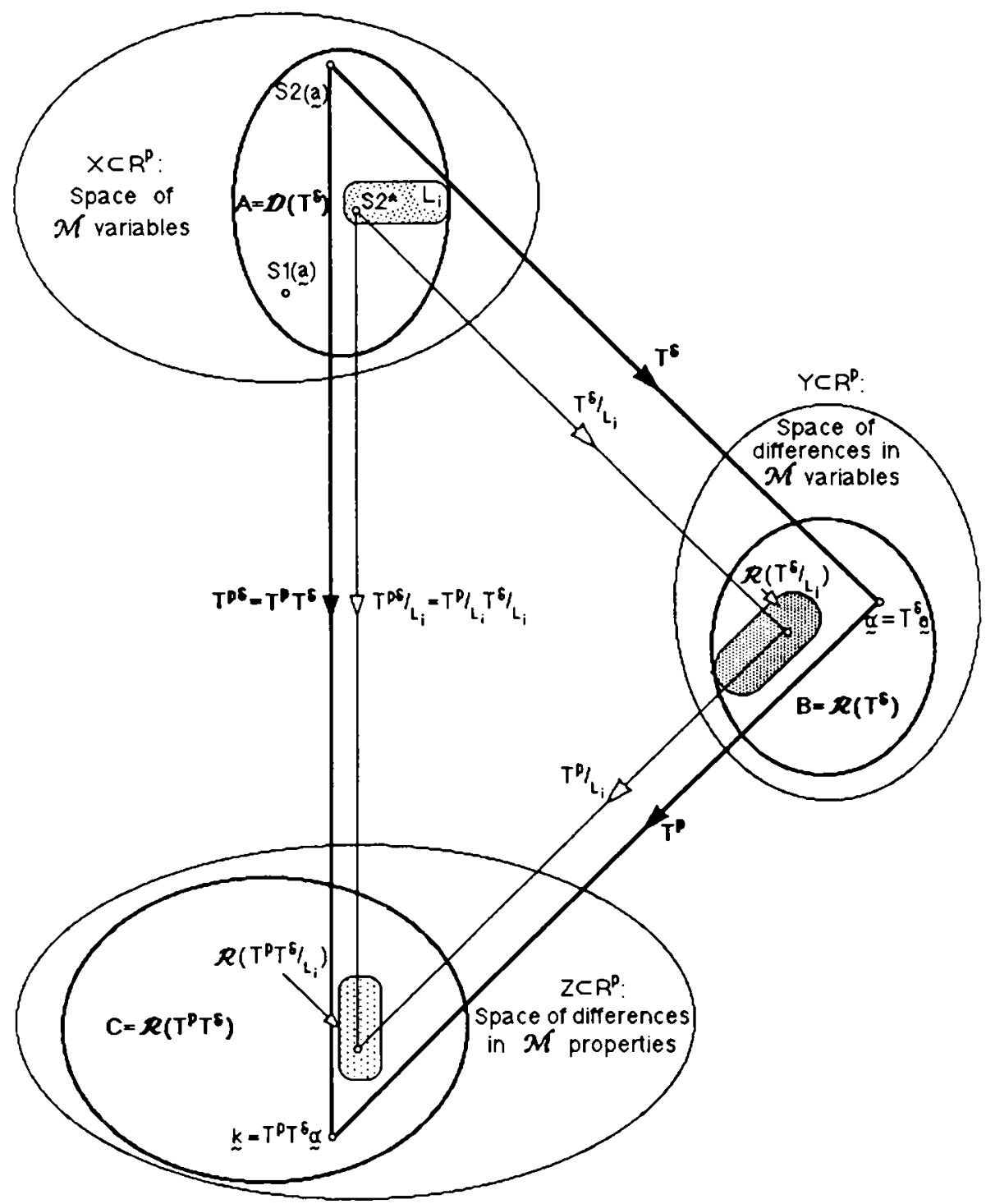

Fig. 2. Injections for redundancy mapping definitions.

be defined uniquely in $A$ or $B$, that is the domain or the range of $T^{\delta} . Y$ is called space of differences in . " variables. In the redundancy definitions in Section 3, and in the numerical applications in Section 6, a fractional change mapping is used instead. It is injective and absolutely equivalent to the difference mapping, so the same symbol is used, $T^{\delta}$.

(D28) A mapping $T^{\delta}$, called fractional change mapping, is defined from $A$ into $Y \subset \cdot \mathscr{P P}^{\mathrm{P}}$ so that: 


$$
\begin{gathered}
T^{\delta}: A=\mathcal{S}\left(T^{\delta}\right) \rightarrow Y \subset \mathscr{p}^{\mathrm{p}} \\
\mathbf{a} \mid \rightarrow T^{\delta} \mathbf{a}=\mathbf{a}
\end{gathered}
$$

and

$$
a_{e}=\frac{a_{e_{2}}-a_{e_{1}}}{a_{e}}, \quad e=1,2, \ldots, p
$$

Here, $Y$ is called space of fractional changes in . $\mathscr{\text { variables. }}$

One more injective mapping is defined between the model variable space $a$ and some structural property (stiffness, mass, load etc.).

(D29) $A$ mapping $T^{\mathrm{p}}$, called structural property mapping, is defined from $B$ into $Z \subset \mathscr{P}^{\mathrm{p}}$ such that

$$
\begin{aligned}
T^{\mathrm{p}}: B=\mathcal{A}\left(T^{\mathrm{p}}\right) & =\mathscr{R}\left(T^{\delta}\right) \rightarrow Z \subset \mathscr{P}^{\mathrm{p}} \\
\boldsymbol{a} \mid & \rightarrow T^{\mathrm{p}} \boldsymbol{a}
\end{aligned}
$$

The range of $T^{\mathrm{p}}$ is $C=\mathscr{h}\left(T^{\mathrm{p}}\right)=\left\{\mathbf{z} \in Z \mid \mathbf{z}=T^{\mathrm{p}} \mathbf{y}\right.$ for some $\left.\mathbf{y} \in \mathcal{S}\left(T^{\mathrm{p}}\right)\right\}$.

Each image of $a$ defines a structural state $S 2$ in terms of some structural property difference from $S 1$ uniquely. The inverse transform $T^{\mathrm{p}^{-1}}$ may not be unique; then $T^{\mathrm{p}}$ would not be injective.

(D30) The composite (or product) mapping $T^{\mathrm{p} \delta}$, is called property difference mapping and is defined from $A$ into $Z$, so that

$$
\begin{aligned}
T^{\mathrm{p} \delta}= & T^{\mathrm{p}} T^{\delta}: A=\mathscr{Y}\left(T^{\mathrm{p} \delta}\right) \rightarrow Z \subset \mathscr{P} \mathrm{p} \\
& \mathbf{a} \mid \rightarrow T^{\mathrm{p} \delta} \mathbf{a}=T^{\mathrm{p}} T^{\delta} \mathbf{a}
\end{aligned}
$$

The range of $T^{\mathrm{p} \delta}$ is $C=\mathscr{R}\left(T^{\mathrm{p} \delta}\right)=\mathscr{R}\left(T^{\mathrm{p}} T^{\delta} 0\right.$

A structural state may be defined uniquely in $B$ or $C$. Now, we can proceed with the definition of redundancy mappings.

(D31) Variable redundancy mapping $\left.T^{\delta}\right|_{L}$, is the restriction of mapping $T^{\delta}: \mathscr{C}\left(T^{\delta}\right) \rightarrow Y$ to a subset $L_{i} \subset \mathscr{C}\left(T^{\delta}\right): L_{i} \rightarrow Y$. In simple terms, a point in $\mathscr{R}\left(\left.T^{\delta}\right|_{L_{i}}\right)$ represents the difference between $\mathrm{S} 2_{i}$ and $\mathrm{S} 1$; and since $\mathrm{S} 2_{i}$ is on the limit surface $L_{i}$, that difference represents redundancy with respect to model variables. Further, since the mapping is an injection, $\mathrm{S} 1$ and the inverse of a redundancy injection can be used to identify $\mathbf{S} 2_{i}$, uniquely. In the literature, several definitions of redundancy are associated with structural properties rather than design variables. Therefore, the following mapping definition is required also.

(D32) Structural property redundancy mapping $\left.T^{\mathrm{p} \delta}\right|_{L_{i}}$, is the restriction of mapping $T^{\mathrm{p} \delta}: \mathcal{C}\left(T^{\mathrm{p} \delta}\right) \rightarrow Z$ to a subset $L_{i} \subset \mathcal{L}\left(T^{\mathrm{p} \delta}\right): L_{i} \rightarrow Z$.

Again, in simple terms, a point in $\mathscr{R}\left(\left.T^{\mathrm{p} \delta}\right|_{L_{i}}\right)$ represents the difference between $S 2_{i}$ and $\mathrm{S} 1$; and since $S 2_{i}$ is on the limit surface $L_{i}$, that difference represents redundancy with respect to some structural property (e.g. 
stiffness, mass, load). If $T^{\mathrm{p} \delta}$ is injective, design will be possible because $\left.T^{\mathrm{p} \delta}\right|_{L_{i}}$ will be injective.

(D33) $\mathscr{R}\left(\left.T^{\delta}\right|_{L_{i}}\right) \subset B$ is called the model variable redundancy space.

(D34) $\mathscr{R}\left(\left.T^{\mathrm{p} \delta}\right|_{L_{i}}\right) \subset C$ is called the model property redundancy space.

In practice, if the appropriate numerical tools (computer codes) are not available redundancy mappings will represent too much information for comparison of structures with respect to redundancy. Thus, various redundancy measures are defined and redundancies are computed. A redundancy measure is a function and redundancy is its numerical value. A variety of those can be found in the literature (see Section 1.1). All can be defined either in the model variable redundancy space $\mathscr{R}\left(\left.T^{\delta}\right|_{L_{i}}\right)$ or the model property redundancy space $\mathscr{R}\left(\left.T^{\mathrm{p} \delta}\right|_{L_{i}}\right)$ by inducing a metric by a norm in those spaces ${ }^{39}$. In simple terms, by making the above two spaces normed spaces, redundancy measures can be defined.

(D35) Redundancy function or redundancy measure is a norm defined in a redundancy vector space. Thus, a redundancy measure can be defined in terms of model variables (e.g. geometry, load) in the $Y$ space, or model properties (e.g. stiffness, mass) in the $Z$ space. Obviously, the relation between $S 1$ and $S 2_{i}$ as measured by a norm may not be unique and will result in loss of design information. To produce a design from $\mathrm{S} 1$ and a redundancy function, additional information, e.g. an optimality criterion, will be needed.

(D36) Redundancy is the numerical value of a redundancy function.

Finally, the concepts of invariancy and consistency are introduced.

Proposition 1: A definition of redundancy is invariant with respect to model variables. Two issues are important in this proposition. First, it is the definition of a mapping or a function that is invariant, not a numerical value; and second, that a definition is invariant with respect to $a_{e}$ but may depend on $\mathscr{H}$. That is, all definitions based on PAR will not depend on factors F1-F6. Their corresponding norms, however, may have values depending on the grid of $\mathscr{H}$.

Proposition 2: Consistent definitions of redundancy are only those that preserve uniqueness in definition of $\mathbf{S} 2_{i}$.

Therefore, only injective difference mappings of the $T^{\delta}$ or $T^{\mathrm{p} \delta}$ kind are consistent.

\section{INVARIANT CONSISTENT REDUNDANCY INJECTIONS}

Assuming that a satisfactory model $\mathscr{H}$ for any structure can be produced by the FE method, and that the code used can predict accurately the response of that structure, all definitions of redundancy provided 
hereafter are invariant. A redundancy definition would not be invariant with respect to a variable (e.g. load, geometry, or material variable), if that variable could not be included in $\mathscr{U}$ and special treatment of that variable would be required depending on the structure. In such a case, FE analysis would not be satisfactory either. In conclusion, PAR brings the level of sophistication of structural computations in redundancy analysis up to par with that of practical structural analysis for large scale continuous or discrete structures.

Several consistent (injective) redundancy mappings are provided below. The geometric redundancy mapping in Section 3.2 is of the $\left.T^{\delta}\right|_{L_{i}}$ kind; the rest are of the $\left.T^{\delta \mathrm{p}}\right|_{L_{i}}$ kind.

\subsection{Redundancy in structural properties}

Let $[k]$ and $[m]$ be the global mass and stiffness matrices of. I. The following perturbation relations between $\mathrm{S} 1$ and $\mathrm{S} 2$ are introduced.

$$
\begin{gathered}
{\left[k^{\prime}\right]=[k]+[\Delta k]} \\
{\left[m^{\prime}\right]=[m]+[\Delta m]}
\end{gathered}
$$

where unprimed and primed quantities pertain to $S 1$ and $S 2$, respectively, and prefix $\Delta$ indicates difference between the two states. $S 2$ is uniquely defined by $S 1$ and $[\Delta k]$ and $[\Delta m]$. Note that $[\Delta k]$ and $[\Delta m]$ may be due to differences in cross-sectional properties like inertia $I$ and area $A$; or due to differences in material properties, like $E$ and $\rho$. In PAR, those differences are treated identically, so hereafter only the former are presented.

\section{Global stiffness and mass redundancy mappings}

If $\mathrm{S} 2$ is an $\mathrm{S} 2_{i}$ state on limit surface $L_{i}$, the mapping of structural state $\mathrm{S} 2_{i}$ is restricted on $L_{i}$, and is a $\left.T^{\delta \mathrm{p}}\right|_{L_{i}}$ mapping because, according to eqns (7) and (8), it defines structural properties $\left[k^{\prime}\right]$ and $\left[m^{\prime}\right]$ uniquely. It is not an injective mapping, however, because the inverse mapping is not unique. This is the case, because $\left[k^{\prime}\right]$ and $\left[m^{\prime}\right]$ are global matrices and cannot be used to define $\mathrm{S} 2_{i}$ uniquely. That is, the global $([\Delta k],[\Delta m])$ domain can be used along with $([k],[m])$ to define $\left(\left[k^{\prime}\right],\left[m^{\prime}\right]\right)$ using eqns (7) and (8) but does not provide adequate information for element design. To achieve that goal we have to go to the FE element level and preserve element connectivity in. $/$.

\section{Element stiffness and mass redundancy injections}

Let $p$ properties of elements or groups of elements differ between structural states $\mathrm{S} 1$ and $\mathrm{S} 2_{i}$. Several elements may be linked together in 
one group and modeled by the same variables. If there is confidence that all elements in a substructure have been manufactured to the same specifications, are subjected to similar environmental loads, and have identical deterioration in time, such linking would be desirable and necessary. Each element or group of elements in $\mathrm{S} 1$ or $\mathrm{S} 2_{i}$ may differ in more than one property. Then, the redundancy mapping $([\Delta k],[\Delta m])$ in global stiffness and mass matrices can be expressed as summation of redundancies in each element or group of elements as

$$
\begin{aligned}
& {[\Delta k]=\sum_{e=1}^{p} \Delta k_{e}=\sum_{e=1}^{p}\left[k_{e}\right] \alpha_{e}} \\
& {[\Delta m]=\sum_{e=1}^{p} \Delta m_{e}=\sum_{e=1}^{p}\left[m_{e}\right] \alpha_{e}}
\end{aligned}
$$

where $\left[k_{e}\right]$ and $\left[m_{e}\right]$ are the stiffness and mass matrices of the $e$ th element or group of elements, $\alpha_{e}, e=1,2, \ldots, p$. Several $\alpha_{e} s$ may refer to the same element but different properties like bending, torsion, stretching, etc. For example, in linear elastic beam bending

$$
\begin{gathered}
{\left[k_{e}^{\prime}\right]=\frac{E I^{\prime}}{l^{3}}\left[k_{B_{e}}\right]=\left[k_{e}\right]+\left[\Delta k_{e}\right]=\frac{E I}{l^{3}}\left[k_{B_{e}}\right]+\left[\Delta k_{e}\right]} \\
{\left[\Delta k_{e}\right]=\frac{E I}{l^{3}} \alpha_{e}\left[k_{B_{e}}\right]} \\
{\left[m_{e}^{\prime}\right]=\rho A^{\prime} l\left[m_{B_{e}}\right]=\left[m_{B_{e}}\right]+\left[\Delta m_{e}\right]=\rho A l\left[m_{B_{e}}\right]+\left[\Delta m_{e}\right]} \\
{\left[\Delta m_{e}\right]=\rho A l \alpha_{e}\left[m_{B_{e}}\right]}
\end{gathered}
$$

in linear elastic beam torsion

$$
\begin{gathered}
{\left[k_{e}^{\prime}\right]=\frac{G J^{\prime}}{l}\left[k_{T_{e}}\right]=\left[k_{e}\right]+\left[\Delta k_{e}\right]=\frac{G J}{l}\left[k_{T_{e}}\right]+\left[\Delta k_{e}\right]} \\
{\left[\Delta k_{e}\right]=\frac{G J}{l} \alpha_{e}\left[k_{T_{e}}\right]}
\end{gathered}
$$

in linear elastic beam stretching

$$
\left[k_{e}^{\prime}\right]=\frac{T}{E A}\left[k_{S_{e}}\right]=\left[k_{e}\right]+\left[\Delta k_{e}\right]=\frac{T}{E A}\left[k_{S_{e}}\right]+\left[\Delta k_{e}\right]
$$

and

$$
\left[\Delta k_{e}\right]=\frac{T}{E A} \alpha_{e}\left[k_{S_{e}}\right]
$$


The above equations define a mapping of the type $\left.T^{\delta \mathrm{p}}\right|_{L_{i}}$ as the global stiffness and mass redundancy mapping does. In addition, there is adequate information for structural design of elements. That is, state $\mathrm{S} 2$ can be defined uniquely from S1 and element mappings. Therefore, element mappings, as defined by eqns (12), (14), (16), (18) are injective and will be called hereafter element redundancy injections (D37). For example, if $\mathrm{S} 2$ is an $\mathrm{S} 2_{i}$ state and $L_{i}$ is the global buckling limit state, then $\left[\Delta k_{e}\right], e=1,2, \ldots, p$, is an injection representing element $e$ redundancy towards global buckling. In dynamic failure, element redundancy injections are defined by both stiffness and mass injections $\left[\Delta k_{e}\right]$ and $\left[\Delta m_{e}\right]$.

It should be reminded at this point, that LEAP theory, as presently implemented in code RESTRUCT, ${ }^{38}$ produces $\left[k^{\prime}\right]$ and $\left[m^{\prime}\right]$ matrices that correspond to a real structure. In methods with objective similar to those of LEAP theory, this may not be the case or only small perturbations may be allowed as explained in the review in reference ${ }^{33}$.

\subsection{Geometric redundancy}

$\left[\Delta k_{e}\right]$ and $\left[\Delta m_{e}\right]$ redundancy injections define uniquely the structural design of elements of $S 2_{i}$ but not necessarily their geometry. For example, a plate element of $S 2_{i}$ is uniquely defined by $\left[k_{e}\right]$ of $S 1$ and a redundancy injection $\left[\Delta k_{e}\right]$ because there is a one-to-one relation between moment of inertia $I$ and plate thickness $t$. This is not the case for a beam of rectangular cross-section where $I=b h^{3} / 12(b=$ breadth and $h=$ height $)$. As another example, consider $\left[\Delta k_{e}\right]$ and $\left[\Delta m_{e}\right]$ redundancy injections for a beam element. If the beam cross-section is rectangular it is defined uniquely by those injections. For an I-beam, however, those structural property injections (stiffness and mass) are not geometric injections. Below, examples of geometric injections (D38), that is of the type $\left.T^{\delta}\right|_{L_{i}}$ implemented in RESTRUCT are defined.

For a rectangular beam cross-section of height $h$ and width $b$, the following relations can be derived for its moment of inertia $I$ and area $A$ : If $\mathrm{S} 1$ and $\mathrm{S} 2_{i}$ differ only in $b$, we will have

$$
\begin{aligned}
& I^{\prime}=\frac{b^{\prime} h^{3}}{12}=\frac{b\left(1+a_{b}\right) h^{3}}{12}=\left(1+a_{b}\right) I \\
& A^{\prime}=b^{\prime} h=b\left(1+a_{b}\right) h=\left(1+\alpha_{b}\right) A
\end{aligned}
$$

If $\mathrm{S} 1$ and $\mathrm{S} 2_{i}$ differ only in $h$, we will have

$$
I^{\prime}=\frac{b h^{\prime 3}}{12}=\frac{b h^{3}\left(1+\alpha_{h}\right)^{3}}{12}=\left(1+\alpha_{h}\right)^{3} I
$$




$$
A^{\prime}=b h^{\prime}=b h\left(1+a_{h}\right)=\left(1+\alpha_{h}\right) A
$$

If $\mathrm{S} 1$ and $\mathrm{S} 2_{i}$ differ both in $b$ and $h$, we will have

$$
\begin{gathered}
I^{\prime}=\frac{b^{\prime} h^{\prime 3}}{12}=\frac{b\left(1+\alpha_{b}\right) h^{3}\left(1+\alpha_{h}\right)^{3}}{12}=\left(1+\alpha_{b}\right)\left(1+\alpha_{h}\right)^{3} I \\
A^{\prime}=b^{\prime} h^{\prime}=b\left(1+a_{b}\right) h\left(1+\alpha_{h}\right)=\left(1+a_{b}\right)\left(1+a_{h}\right) A
\end{gathered}
$$

Depending on the relation between the number of element structural property variables $n_{p}(A, I$, torsional stiffness $J$, etc.) and the number of element geometric variables $n_{g}(b, h$, etc.) it may or may not be possible to define mappings and injections. Three different problems may arise.

(P1) If $n_{g}=n_{p}$ an element geometric redundancy injection can be defined. Any one of eqns (19)-(22) provide examples of such injection because in all four cases $n_{p}=n_{g}=1$.

(P2) If $n_{g}>n_{p}$ a noninjective element geometric mapping can be defined. Equations (23) or (24) define noninjective mappings because in both cases $n_{g}=2, n_{p}=1$. In RESTRUCT ${ }^{38}$ a solution of this underdetermined problem is achieved by introducing an optimality criterion.

(P3) If $n_{g}<n_{p}$ the problem is overdetermined and even a mapping cannot be established. This is the case for a plate element of unit length and thickness $t$, where

$$
\begin{aligned}
& A^{\prime}=t^{\prime}=\left(1+\alpha_{t}\right) t=\left(1+\alpha_{t}\right) A \\
& I^{\prime}=\frac{t^{\prime 3}}{12}=\frac{\left(1+\alpha_{t}\right)^{3} t^{3}}{12} \cong\left(1+\alpha_{t}\right)^{3} I
\end{aligned}
$$

In RESTRUCT a solution is provided by minimizing the error in the constraints of the overdetermined problem. Note that the inverse of problems (P2) and (P3), are problems (P3) and (P2), respectively.

\subsection{Performance redundancy}

Material property and geometric redundancies (design redundancies), as defined in Sections 3.1 and 3.2, a re necessary from the design point of view. In a complete design process, however, they are not sufficient; they must be related to performance or strength redundancies (feature or specification redundancies) in order to achieve direct design without trial and error or repeated FE analyses. Our goal in this and the next section is to define mappings relating design to specification redundancies. The latter will be related to failure criteria in Section 4. Obviously, those may or may not be injective depending on the number of design variables and specification particulars. For example, many 
structures modeled by ." may have the same first natural frequency when more than one design variable is used in . $/$ (problem P2). Then an optimality criterion will be required to select an $\mathrm{S} 2$ state and make the mapping injective. If the relation between specifications and design variables is unique, the mapping will be injective (Problem P1). If the problem is overdetermined (more specifications than design variables) there can be no conventional mapping (P3). Minimization of error in satisfaction of specifications is implemented in RESTRUCT but the produced S2 even if unique is not considered to be the product of conventional mapping.

Two forms of performance redundancy have been implemented in RESTRUCT, modal dynamics and static deflections. Strength may also be considered as performance; two forms of strength redundancy presently under development are presented separately in Section 3.4.

\section{Modal dynamics}

Frequencies and mode shapes a re important because they can be related to service or survival failure. The following perturbation relations between $\mathrm{S} 1$ and $\mathrm{S} 2$ are introduced.

$$
\begin{gathered}
{\left[\omega^{\prime 2}\right]=\left[-\omega^{2}\right]+\left[-\Delta\left(\omega^{2}\right)\right]} \\
{\left[\phi^{\prime}\right]=[\phi]+[\Delta \phi]}
\end{gathered}
$$

where $[\phi]=\left[\{\psi\}_{1},\{\psi\}_{2}, \ldots,\{\psi\}_{n}\right]$, is the matrix of eigenvectors of $S 1$ and $\left[-\omega^{2}\right]$ is the diagonal matrix of the corresponding eigenvalues. $[\Delta \phi]$ and $\left[-\Delta\left(\omega^{2}\right)_{-}\right]$are redundancy expressions. To develop redundancy mappings or injections we must relate those to design redundancies. Above all we want to make sure that all definitions of redundancy, whether introduced here or appearing in previous literature, are consistent. The appropriate relations are provided by the free vibration equilibrium of $\mathrm{S} 1$

$$
\left([k]-\omega_{j}^{2}[m]\right)\{\psi\}_{j}=\{0\} \text { for } j=1,2, \ldots, n
$$

where $n$ eigenvalues $\omega_{j}, j=1,2, \ldots, n$ satisfy eqn (30)

$$
\operatorname{det}\left([k]-\omega_{j}^{2}[m]\right)=0
$$

In eqn (29), added mass is included in $[m]$ and damping may be included only in Rayleigh's form. The uncoupled modal equations are

$$
\left[-K_{-}\right]=\left[-M_{-}\right]\left[\omega^{2}-\right]
$$

where $\left[K_{-}\right]$and $\left[M_{-}\right]$are the generalized stiffness and mass matrices. Similar equations (with primed quantities) hold for S2. Equations (29)(31) provide the relation between design (structural properties, geometry) and performance $\left(\omega_{j},\{\psi\}_{j}\right)$ through eqns (9) and (10). The counterpart 
relations to (29)-(31) for S2 provide relations between design and performance for S2. This process is part of PAR. LEAP theory then can provide the relation between S1 and S2 which may be a mapping, an injection, or overdetermined (see problem P1-P3 in Section 3.2). These relations are the general perturbation equations providing failure equations as shown in Section 4.

\section{Static deflection}

Static deflection can also be related to service or survival failure. Between $\mathrm{S} 1$ and $\mathrm{S} 2$ the following perturbation relations may be introduced

$$
\left\{u^{\prime}\right\}=\{u\}+\{\Delta u\}
$$

and

$$
\left\{f^{\prime}\right\}=\{f\}+\{\delta f\}
$$

where the governing equation for static FE analysis is

$$
[k]\{u\}=\{f\}
$$

A similar (primed) equation holds for S2. Again, eqn (34) and its counterpart for $\mathrm{S} 2$ relate design variables to performance particulars through eqn (9). The relations between $S 1$ and $S 2$ are developed in Section 4 . They are the corresponding general perturbation equations and may provide a mapping, an injection, or an overdetermined relation.

\subsection{Strength redundancy}

Two forms of strength redundancy are considered in our research; stress and buckling redundancy. Neither is reported in this paper. The former has already been implemented in RESTRUCT. Buckling is under development. Both require extensive presentation and will be published separately. Suffice to present here the basic relations of PAR which LEAP theory uses to define S2 by post processing S1 FE a nalysis results.

\section{Stress redundancy}

The following perturbation relations between $\mathrm{S} 1$ and $\mathrm{S} 2$ are introduced

$$
\begin{aligned}
& \left\{\sigma^{\prime}\right\}=\{\sigma\}+\{\Delta \sigma\} \\
& {\left[S^{\prime}\right]=[S]+[\Delta S]}
\end{aligned}
$$

where

$$
\begin{aligned}
& \{\sigma\}=[S][k]^{-1}\{f\} \\
& {[S]=[G][D][N]}
\end{aligned}
$$


and $[G],[D],[N]$ are the stress-strain, strain-displacement, and shape function matrices, respectively. Expressing change in the global matrix as sum of all element changes we have

$$
[\Delta S]=\sum_{e=1}^{p}\left[\Delta S_{e}\right]=\sum_{e=1}^{p}\left[S_{e}\right] \alpha_{e}
$$

As in the case of stiffness and mass, global mapping is not injective, in general. At the element level the redundancy mapping is injective. Redundancy in stress $\{\Delta \sigma\}$, however, could be a mapping, an injection or overdetermined.

\section{Buckling redundancy}

The governing equation of buckling is

$$
\left(\left[k_{o}\right]+\left[k_{\sigma}\right]\right)\left\{\psi_{b}\right\}=\{0\}
$$

where $\left[k_{o}\right]$ and $\left[k_{\sigma}\right]$ are the small displacement and initial geometric stiffness matrices, respectively. Again, perturbation relations can be introduced to relate $\mathrm{S} 1$ to $\mathrm{S} 2$ at the performance level, critical loads and buckling modes, or at the element level.

To summarize, the authors have so far taken the first step in PAR (see Section 2.2). That is, they have developed perturbation relations between $\mathrm{S} 1$ and $\mathrm{S} 2$ and have provided invariant definitions for redundancy mappings and injections. The latter are consistent and can be used in design. The second step in PAR is to produce failure equations in Section 4 ; the third step is to use LEAP theory to calculate to failure points and the corresponding redundancy injections without trial and error and without repeated FE analyses.

\section{FAILURE EQUATIONS}

The second step in PAR consists of defining a global failure criterion, quantifying it if necessary, and derive a global failure equation for the structure. In the literature, lack of global failure equations appears to be a major obstacle in computation of redundancy and reliability of large scale structures ${ }^{20,40}$. General perturbation equations, like those derived in this section, provide global failure equations. The procedure involves the following sequential actions

(A1) Select a failure criterion (see D18).

(A2) Quantify the failure criterion if necessary (see D20).

(A3) Perform the corresponding FE analysis for S1 (see D3). 
(A4) Derive the general perturbation equations expressing $S 2_{i}$ in terms of the failure criterion, and FE analysis data and results for S1.

It should be remembered that all failu re criteria can be related to some quantification of structural performance. For example, a singularity in the buckling stiffness matrix results in global buckling; a zero first natural frequency results in mechanism, etc. It should be noted that even if a local property is quantified to set a service failure criterion, the effect is global and the corresponding failure equation is global; e.g. if $u_{j}=u_{j \max }$ is the failure criterion for a propeller shaft where $j$ is a slope degree of freedom, even though it appears to be a local failure criterion it is indeed a global one since the entire structure deformation contributes to $u_{j}$.

\subsection{Modal dynamic failure}

Following actions, (A1)-(A4) defined above we have the following.

(A1) Select as a failure criterion any one modal response characteristic of S2; say a natural frequency $\omega_{j}$ or some degree of freedom of a mode $\psi_{i j}$ or any combination of the above.

(A2) Quantify that criterion; e.g. $\omega_{1}$ should have a lower limit of $\omega_{1}^{\prime}$. From the practical point of view this means that a structure, say an offshore tower, is assumed to have deteriorated to the point of failure when $\omega_{1}$ has reached its lower limit.

(A3) It is assumed then, that the modal dynamic FE analysis of $\mathrm{S} 1$ is performed to determine the modal dynamics (natural frequencies and mode shapes) of $S 1$.

(A4) Relating eqn (31), its counterpart for $\mathrm{S}_{i}$, and perturbation relations (7)-(10), (27), (28), we derive the general perturbation equations in terms of the $\alpha_{e} s$ as

$$
\begin{aligned}
& \sum_{e=1}^{p}\left(\left\{\psi^{\prime}\right\}_{i}^{T}\left[k_{e}\right]\left\{\psi^{\prime}\right\}_{i}-\omega_{i}^{\prime 2}\left\{\psi^{\prime}\right\}_{i}^{T}\left[m_{e}\right]\left\{\psi^{\prime}\right\}_{i}\right) a_{e} \\
& =\omega_{i}^{\prime 2}\left\{\psi^{\prime}\right\}_{i}^{T}[m]\left\{\psi^{\prime}\right\}_{i}-\left\{\psi^{\prime}\right\}_{i}^{T}[k]\left\{\psi^{\prime}\right\}_{i} \\
& \sum_{e=1}^{p}\left\{\psi^{\prime}\right\}_{j}^{T}\left[k_{e}\right]\left\{\psi^{\prime}\right\}_{i} \alpha_{e}=-\left\{\psi^{\prime}\right\}_{j}^{T}[k]\left\{\psi^{\prime}\right\}_{i} \\
& \sum_{e=1}^{p}\left\{\psi^{\prime}\right\}_{j}^{T}\left[m_{e}\right]\left\{\psi^{\prime}\right\}_{i} \alpha_{e}=-\left\{\psi^{\prime}\right\}_{j}^{T}[m]\left\{\psi^{\prime}\right\}_{i} \\
& \text { for } i=1,2, \ldots, n, \quad j=i+1, i+2, \ldots, n
\end{aligned}
$$


Recall that the $\alpha_{e} s$ represent an element geometric redundancy injection. Equation (41) represents the $n$ diagonal terms in the counterpart of eqn (31) for $S 2_{i}$, that is the Rayleigh quotients for $\omega_{i}^{\prime 2}$. Equations (42) and (43) are equivalent to the orthogonality conditions of the $S 2_{i}$ modes $\left\{\psi^{\prime}\right\}_{i}$ with respect to $\left[k^{\prime}\right]$ and $\left[m^{\prime}\right]$. Theoretically, orthogonality of modes with respect to one of $\left[k^{\prime}\right]$ or $\left[m^{\prime}\right]$ implies orthogonality with respect to the other. Numerically, however, both conditions must be forced if $\left\{\psi^{\prime}\right\}_{j}$, $j=1,2, \ldots, n$, are to represent modes of a real structure. In the LEAP process, eqns (41)-(43) are imposed in $S 2_{i}$. It should be noted that these are the global failure equations and are implicit expressions of $a_{e} s$. Their solution represents challenge $C 5$ addressed by LEAP theory in Section 5 .

Three issues are worth noting.

(i) A global structural collapse can be quantified by setting $\omega_{1}^{\prime}=0$. Then eqn (41) represents the global failure equation. Equations (42) and (43) are the admissibility conditions forced to ensure that the $S 2_{i}^{*}$ (actual failure point on limit surface $i$ ) calculated by LEAP represents a real structure.

(ii) $\mathrm{S} 2_{i}^{*}$ is to be defined directly from the failure equations and the term 'failure mode identification' is not applicable in PAR because the structure depends continuously on the $\alpha_{e} s$.

(iii) Element failure must be detected as it occurs and accommodated by reconfiguration. Yet, state $S 1$ need not be redefined (see D2) until the LEAP algorithm (see Section 5) loses its accuracy.

\subsection{Static deflection failure}

Repeating the procedure followed in Section 4.1 we have:

(A1) Select as failure criterion one or more dofs of $\{u\}$, the static deflection vector in eqn (34).

(A2) Quantify that criterion; e.g. $u_{i}$ should have an upper bound $u_{i \max }$, that is $u_{i}<u_{i \max }$.

(A3) Perform static FE analysis of $\mathrm{S} 1$ to determine the static response of $\mathrm{S} 1$.

(A4) Relating eqn (34), its counterpart for $\mathrm{S}_{2}$, and perturbation relations (32), (33) we derive the following general perturbation equation

$$
u_{i}^{\prime}=\sum_{m=1}^{n_{r}}\left(\frac{\phi_{i m}^{\prime} A_{m}}{B_{m}}\right)-\sum_{e=1}^{p}\left(\sum_{m=1}^{n_{r}} \frac{\phi_{i m}^{\prime} A_{m}}{B_{m}^{2}} C_{m e}\right) a_{e}
$$

where

$$
A_{m}=\sum_{j=1}^{n}\left(\phi_{j m}^{\prime} f_{j}^{\prime}\right), \quad B_{m}=\left\{\psi^{\prime}\right\}_{m}^{T}[k]\left\{\psi^{\prime}\right\}_{m}, \quad C_{m e}=\left\{\psi^{\prime}\right\}_{m}^{T}\left[k_{e}\right]\left\{\psi^{\prime}\right\}
$$


Equation (44) was derived by linearizing only the explicit dependence on $a_{\varepsilon} s$, for consistency with the prediction phase of the LEAP algorithm presented in Section 5. Derivation of eqn (44) is achieved by a series expansion of $\left\{u^{\prime}\right\}$ in terms of the unknown modes $\left\{\psi^{\prime}\right\}_{j}, j=1,2, \ldots, n^{25}$. Thus, inversion of matrix $\left[k^{\prime}\right]$ is avoided. Equation (44) is an implicit expression of the $\alpha_{e} s$ and for a given $u_{i \max }$ it provides a global failure equation. Again, recall that the $\alpha_{e} s$ represent an element geometric redundancy injection. Whether the problem reduces to P1, P2, or P3 (see Section 3.2) depends on the number of $u_{i \max } s$ (failure criteria) specified and the $\alpha_{e} s$.

\subsection{Other failure equations}

For any two-state problem that satisfies the LEAP principle, general perturbation equations can be derived. Those are the global failure equations. For stresses we have

$$
\begin{aligned}
\{\Delta \sigma\}=-\{\sigma\}+ & \left([S]+\sum_{e=1}^{p}\left[S_{e}\right] \alpha_{e}\right) \\
& {\left[\sum_{m=1}^{n} \frac{\phi_{i m}^{\prime} A_{m}}{B_{m}}-\sum_{e=1}^{p}\left(\sum_{m=1}^{n} \frac{\phi_{i m}^{\prime} A_{m}}{B_{m}^{2}} C_{m_{e}}\right) \alpha_{e}\right] }
\end{aligned}
$$

and for global Euler buckling we have

$$
\begin{aligned}
& \sum_{e=1}^{p}\left\{\psi_{b}^{\prime}\right\}_{i}^{T}\left(\left[k_{c_{e}}\right]-P_{i}^{\prime}\left[k_{\sigma_{o} e}\right]\right)\left\{\psi_{b}^{\prime}\right\}_{i} \alpha_{e}=\left\{\psi_{b}^{\prime}\right\}_{i}^{T}\left(P_{i}^{\prime}\left[k_{\sigma_{o}}\right]-\left[k_{c}\right]\right)\left\{\psi_{b}^{\prime}\right\}_{i}, \\
& \text { for } i=1,2, \ldots, n \\
& \quad \sum_{e=1}^{p}\left\{\psi_{b}^{\prime}\right\}_{j}^{T}\left[k_{c_{e}}\right]\left\{\psi_{b}^{\prime}\right\}_{i} \alpha_{e}=-\left\{\psi_{b}^{\prime}\right\}_{j}^{T}\left[k_{c}\right]\left\{\psi_{b}^{\prime}\right\}_{i} \\
& \sum_{e=1}^{p}\left\{\psi_{b}^{\prime}\right\}_{j}^{T}\left[k_{\sigma_{e} e}\right]\left\{\psi_{b}^{\prime}\right\}_{i} \alpha_{e}=-\left\{\psi_{b}^{\prime}\right\}_{j}^{T}\left[k_{\sigma_{o}}\right]\left\{\psi_{b}^{\prime}\right\}_{i} \\
& \text { for } i=1,2, \ldots, n, \quad j=i+1, i+2, \ldots, n
\end{aligned}
$$

where $\left[k_{c}\right]=\left[k_{o}\right]-\left[k_{\sigma F}\right]$, and $k_{\sigma F}$ includes the body force. Stresses and buckling are not used in this paper so no further explanations are provided. It should be pointed out, however, that those global failure equations are implicit with respect to the $\alpha_{e} s$ and are similar in nature with the modal dynamic and the static deflection global failure equations. 
Finally, it should be noted that any number of failure criteria can be used simultaneously. General perturbation equations will produce the corresponding global failure equations. Such equations include all model . $\mathscr{H}$ variables and define the safe domain of the structure. Further, the $\alpha_{e} s$ can be computed by the LEAP algorithm (see Section 5) providing the element geometric redundancy injection required in design.

In conclusion, up to now we have defined invariant and consistent redundancy mappings, injections, and measures and we have completed the first two steps in PAR. Next, LEAP theory is used to develop numerical algorithms to solve the problem efficiently.

\section{FAILURE POINT}

In this Section, the LEAP theory solution to the problem of failure point identification is presented. The problem definition is first presented in Section 5.1 and the present status of LEAP theory in solution of the redundancy problem is restated. The problem is formulated and solved in Sections 5.2 and 5.3.

\subsection{Problem definition}

Figure 3 shows a simplified space of model variables after an element difference (not redundancy) injection has been performed as per eqns (3), (4). There are two fractional difference variables $\alpha=\left(\alpha_{1}, \alpha_{2}\right)$; two failure equations $g_{i}(a)=0$ and $g_{j}(a)=0$; two individual failure points $S 2_{i}^{*}\left(\boldsymbol{\alpha}_{i}^{*}\right)$ and $\mathrm{S} 2_{j}^{*}\left(\boldsymbol{\alpha}_{j}^{*}\right)$; one joint failure point $\mathrm{S} 2_{i j}^{*}\left(\boldsymbol{\alpha}_{i j}^{*}\right)$. The failure equations are provided by combining failure criteria quantifications with general perturbation equations like (41)-(43) for modal dynamics; (44), (45) for static failure; (46) for static stresses (47)-(49) for global buckling. State $\mathrm{Sl}$ is located at $\alpha=0$ and one FE analysis has been performed on S1 for each type of failure criterion considered. In general, failure points differ significantly from $\mathrm{S} 1$ and they are to be identified by LEAP without additional $F E$ analyses or trial and error. In this example, to identify the joint failure point $S 2_{i j}^{*}$ a problem of type P2 (see Section 3.2) must be solved because the point is unique. To identify $S 2_{i}^{*}$ or $S 2_{j}^{*}$ an optimality criterion is needed; in our computations the Eucledian norm is defined as the redundancy function (measure) and minimized subject to $g_{i}(\alpha)=0$ or $g_{j}(\alpha)=0$, respectively. The meaning of this process is that the difference between $\mathrm{S} 1$ and the failure point is assumed to be minimal. Other optimality criteria (like minimum weight difference) may be used.

The global failure equations derived in PAR are implicit and difficult 


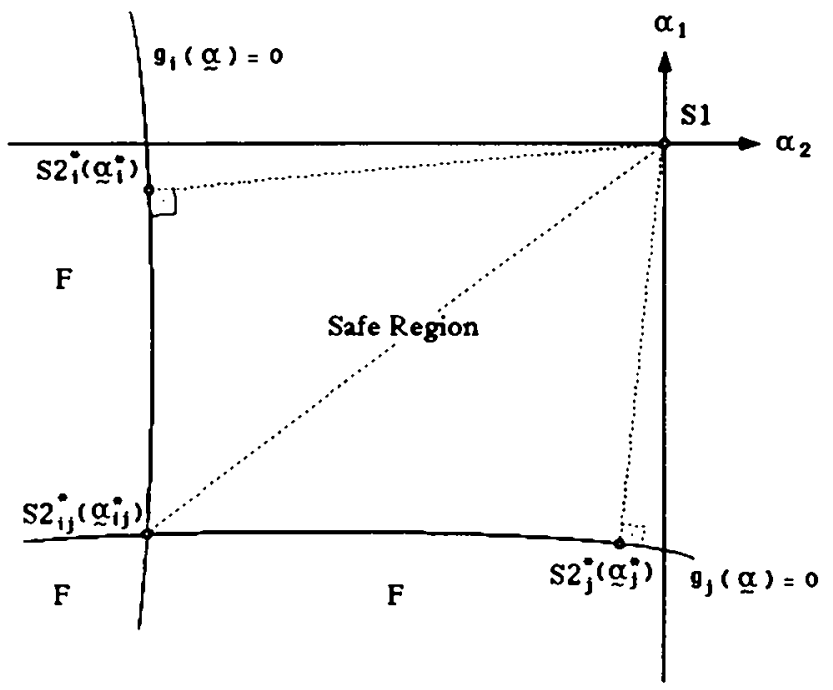

Fig. 3. Failure surfaces; individual $\left(a_{i}^{*}, a_{j}^{*}\right)$ and joint $\left(a_{i j}^{*}\right)$ limit state points.

to solve. LEAP theory does not enumerate paths to failure to define a failure point; it computes the optimal point subject to a limit state equation. The term 'Failure Mode Identification' is replaced in PAR by Failure Point Identification' due to the continuity of the failure equation with respect to $\alpha$. The discontinuity occurring when an element breaks is part of the accommodation/reconfiguration process occurring as a LEAP algorithm optimizes its way from $S 1$ to the failure point. In PAR, the 'Failure Mode' depends on so many continuous variables and their randomness that it is of no practical use to trace it. In LEAP theory, it is produced as a by-product of the definition of a failure point.

The present status of LEAP theory as implemented in RESTRUCT is as follows. Natural frequency, mode shape, and static deflection global failure criteria are turned into global failure equations by developing the corresponding general perturbation equations. Individual and joint failure points are defined without trial and error by postprocessing data of S1 analyses performed by MSC/NASTRAN.V64. Structures of up to 1254 dofs have been analyzed successfully ${ }^{30}$. Stress and buckling failure equations are not implemented in the numerical applications in Section 6. Stress general perturbation equations have been implemented in RESTRUCT. Only geometric and material variables are used in our applications even though the general perturbation equations involve all model variables. Element failure detection based on stress limits is simple due to eqn (46). The model reconfiguration process is under development. Updating of the limit state equation is easy because LEAP 
keeps track of all finite element matrices $\left[k_{e}\right]$ and $\left[m_{e}\right]$, not just the global matrices $[k]$ and $[m]$. The challenge is in detecting the limits of accuracy of large admissible perturbations. We are in the process of implementing an energy approach which automates the process of defining the limits of accuracy of the LEAP algorithm. Specifically, as elements deteriorate (say corrosion, randomness etc.), as RESTRUCT optimizes its way to the failure point, the extracted modes in the modal expansion (which are updated at each increment) may lose their accuracy. Then S1 must be redefined and a nalyzed by the FE code once more. Even though element failure contributes to the change of the modal basis it is not the main factor. In the rest of this Section the present status of LEAP and RESTRUCT is shown.

\subsection{Problem formulation}

In general, the problem of identifying $S 2_{i}^{*}$ requires optimization to define $S 2_{i}^{*}$ uniquely. Once $S 2_{i}^{*}$ is identified, $\alpha$ becomes $\alpha_{i}^{*}$ and this defines an element redundancy injection. The overall optimization problem which defines the feasibility domain in a multiple failure criterion problem is defined below. Obviously, when individual failure points are to be determined only one failure criterion is to be used at a time.

$$
\text { Minimize }\|\boldsymbol{\alpha}\|_{2} \in \mathfrak{p}^{\mathrm{p}}
$$

subject to $n_{\omega}$ natural frequency failure criteria

$$
\omega_{i}^{\prime 2}=\omega_{i}^{2}+\Delta \omega_{i}^{2}, \quad i=1,2, \ldots, n_{\omega}
$$

$n_{\phi}$ normal mode failure criteria

$$
\phi_{k i}^{\prime}=\phi_{k i}+\Delta \phi_{k i}, \quad \text { number of }(k, i)=n_{\phi}
$$

$n_{u}$ static deflection failure criteria

$$
u_{i}^{\prime}=u_{i}+\Delta u_{i}, \quad i=1,2, \ldots, n_{u}
$$

$n_{\sigma}$ static stress failure criteria

$$
\sigma_{i}^{\prime}=\sigma_{i}+\Delta \sigma_{i}, \quad i=1,2, \ldots, n_{\sigma}
$$

$n_{b}$ global buckling eigenvalues

$$
P_{i}^{\prime}=P_{i}+\Delta P_{i}, \quad i=1,2, \ldots, n_{b}
$$

$n_{\phi b}$ buckling mode failure criteria

$$
\phi_{b_{k i}}^{\prime}=\phi_{b_{k i}}+\Delta \phi_{b_{k t}}, \quad \text { number of }(k, i)=n_{\phi b}
$$


where the left hand sides of eqns (51)-(56) are the quantifications of the failure criteria; $2 p$ lower and upper bounds on geometric variables

$$
-1<\alpha_{e} \alpha_{e}^{-}<\ell_{1 \prime e}<\alpha_{e}^{+}, \quad e=1,2, \ldots, p
$$

and

$$
n_{a}=2 \sum_{i=1}^{n_{\omega}}\left(n_{r}-i\right)=n_{\omega}\left[\left(2 n_{r}-1\right)-n_{\omega}\right]
$$

admissibility constraints (42), (43). In eqns (51)-(56), the right-hand sides are replaced by the corresponding perturbation equations. Our next task is to find individual or joint design points without repeated $\mathrm{FE}$ analyses.

\subsection{Large admissible perturbations in cognate space}

The basic developments of LEAP algorith ms were done for redesign and model correlation ${ }^{25.26 .33}$. Here, only the basic principles are repeated as an algorithm is developed for identification of the failure point, and computation of the element redundancy injection (see D37 in Section 3.1) and the overall structural redundancy.

(a) Starting from S1, the failure point is reached in $N$ increments in a prediction-correction scheme which is summarized in Fig. 4. That is, any quantification of a failure criterion say $\Delta \omega_{1}^{\prime 2}$ in eqn (51), is achieved in $N$ increments, each no larger than 7\%. Thus, the incremental values of $\iota_{e}$, $e=1,2, \ldots, p$, remain small in each increment $\iota$ and

$$
-1<\alpha_{e}^{-}<1 \alpha_{e}<\alpha_{e}^{+}, \quad e=1,2, \ldots, p, \quad \ell=1,2, \ldots, N
$$

where

$$
\iota_{\iota} \alpha_{e}^{-}=-0 \cdot 15, \quad \alpha_{e}^{+}=+0 \cdot 15, \text { and }\left(1+\alpha_{e}\right)=\prod_{l=1}^{N}\left(1+\iota_{\ell}\right)
$$

(b) In the prediction phase of the algorithm in each increment, inadmissible linear perturbations are performed. This part of the algorithm is based on the small perturbation method developed by Stetson $e t$ al. $^{23 .}{ }^{24}$ and improved by Sandström et al. ${ }^{28}$. Incremental modal changes are expressed in terms of the incremental matrix of admixture coefficients $/ \mid c]$, as $[\delta l \phi]=\langle| \phi] / \mid c]^{T}$, where $\iota_{\mathrm{ii}}=0, c_{\mathrm{ij}}, i, j=1,2, \ldots, n_{r}$, $i \neq j$, are small, and $n$, is the number of extracted modes used in the algorithm. Then,

$$
\delta_{\iota} \phi_{k i}=\sum_{j=1, j \neq i}^{n_{r}} \iota \phi_{k j} \iota \mathrm{c}_{\mathrm{ji}}
$$


INCREMENT L

\begin{tabular}{|c|c|c|}
\hline & $\begin{array}{l}\text { Inadmissible } \\
\text { Lineor Prediction }\end{array}$ & $\begin{array}{l}\text { Admissible } \\
\text { Nonlineor Correction }\end{array}$ \\
\hline $\begin{array}{l}\text { Foilure } \\
\text { Criteria }\end{array}$ & $\begin{array}{l}\omega_{i}^{2}, \phi_{k i}^{\prime}, u_{i}^{\cdot}, \sigma_{i}, P_{c r_{i}}^{\prime} \\
(27),(28),(32),(35),(47)\end{array}$ & $\begin{array}{l}\omega_{i}^{.2}, u_{i}^{\prime}, \sigma_{i}^{\prime}, P_{c r_{i}}^{\prime} \\
(27),(32),(35),(47)\end{array}$ \\
\hline $\begin{array}{l}\text { Foilure } \\
\text { Equations }\end{array}$ & $\begin{array}{l}\delta_{l} w_{i}^{2}, \delta_{l} \phi_{k 1}, l^{u} u_{i}, \delta_{l} \sigma_{i}, P P_{e_{i}} ; \\
(62),(61),(44),(46),(47)\end{array}$ & $\begin{array}{l}\delta_{\ell} \omega_{i}^{2}, \ell^{u_{i}}, \delta_{\ell} \sigma_{i}, l P_{c r_{i}} \\
(62),(44),(46),(47)\end{array}$ \\
\hline $\begin{array}{l}\text { RESTRUCT } \\
\text { Dotobose }\end{array}$ & $\begin{array}{l}\delta_{\ell} \omega_{i}^{2}, \delta_{\ell} \Phi_{k i}, \ell u_{i}^{1}, \delta_{\ell} \sigma_{i}, \ell P_{c r_{i}} \\
\ell-1[k], \ell-1\left[m \mid, \ell-1\left[\omega_{-}^{2}\right], \ell-\{\phi]\right. \\
\ell-1(u), \ell-1\left[P_{c r}\right], \ell-1\left[\phi_{b}\right], \ell-1(\sigma)\end{array}$ & $\begin{array}{l}\delta_{\ell} \omega_{i}^{2}, \ell u_{i}^{\prime}, \sigma_{\ell} \sigma_{i}, \ell P_{c r_{i}} \\
\ell-1 \mid k], \ell-1(m], \ell-1\left[\omega^{2}\right], \ell\left[\phi^{\prime}\right], \\
\ell-1(u), \ell-1\left[-P_{c r}\right], \ell\left[\phi_{b}^{j}\right], \ell-1(\sigma)\end{array}$ \\
\hline $\begin{array}{l}\text { Design } \\
\text { Voriables } \\
\text { Admissible } \\
\text { Domoin }\end{array}$ & $\begin{array}{r}\alpha_{\theta, e}=1 \\
n_{\omega}+n_{\phi}+n_{U}+n_{\sigma}+n_{P}+n_{\phi_{b}} ; \\
(51\rangle,\langle 52\rangle,(53),(54),\langle 55\rangle,\langle 56) \\
2 p \text { boun }\end{array}$ & $\begin{array}{l}n_{\omega}+n_{u}+n_{0}+n_{p}+n_{0} \\
\langle 51\rangle,\langle 53\rangle,(54),(55),(58) \\
,(59\rangle\end{array}$ \\
\hline $\begin{array}{l}\text { Solution } \\
\text { Method }\end{array}$ & $\begin{array}{ll}\text { Infinite Solutions: } & \begin{array}{l}\text { Neorest } \\
\text { Doints; }\end{array} \\
\text { No Solution } & \text { No railur } \\
& \text { Minimum }\end{array}$ & $\begin{array}{l}\text { dividual ond joint failure } \\
R_{e} \text { 's within bounds } \\
\text { within ca. bounds: } \\
\text { error solution }\end{array}$ \\
\hline Dutput & $\begin{array}{l}\text { For } L=1 \text {, Cognote Space } \\
\text { Identificotion } \\
\text { Linear predictions } \\
\qquad\left[\phi^{\cdot}\right](61), \ell_{\ell}\left[\phi_{b}\right] ; \\
\alpha_{4}, e=1\end{array}$ & 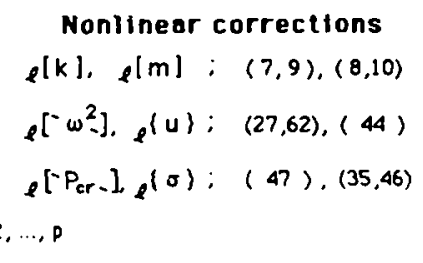 \\
\hline Redundoncy & $\begin{array}{l}\text { Stiffness and Mass Mapping: } \\
\text { Stiffness ond Moss Injection: } \\
\text { Geometry Injection ond Morm : } \\
\text { Performance Mapping: } \\
\text { Strength Mapping: }\end{array}$ & $\begin{array}{l}{[\Delta k],[\Delta m]} \\
{\left[\Delta k_{,}\right],\left[\Delta m_{e}\right], e=1,2, \ldots, p} \\
a_{e}, e=1,2, \ldots, p ;|\underline{q}|_{2} \\
{\left[-\Delta \omega^{2}\right],[\Delta \phi],\{\Delta u\}} \\
{\left[-\Delta P_{c r-}\right],(\Delta \sigma)}\end{array}$ \\
\hline
\end{tabular}

Fig. 4. Algorithm for structural redundancy.

Applying that method to the incremental counterpart of the energy balance eqn (31), diagonal and offdiagonal terms yield, respectively

$$
\begin{gathered}
\delta \omega_{i}^{2}=\frac{1}{\iota M_{i}}\left[\sum_{e=1}^{p}\left(\lambda\{\psi\}_{i}^{T}\left[k_{e}\right] /\{\psi\}_{i}-\iota_{i}^{2} \iota\{\psi\}_{i}^{T}\left[m_{e}\right] \curlywedge\{\psi\}_{i}\right) \alpha_{e}\right] \\
i=1,2, \ldots, n_{\omega}
\end{gathered}
$$




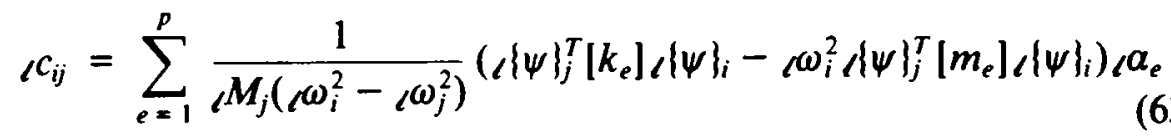

A major advantage of linear perturbation theory is that frequency and modal eqns (62) and (63) are decoupled. A drawback is that linear predictions are inadmissible in the sense that orthogonality conditions with respect to $[k]$ and $[m]$ are satisfied only approximately in the form of eqn (63). In the prediction phase, the incremental counterpart of eqn (44) is used to force static deflection failure criteria. The obvious advantage of eqn (44) is that it provides an explicit expression for static deflections without solving the corresponding static FE problem. That expression, however, depends on the unknown modal basis $\left[\phi^{\prime}\right]$ and on the existing force $\left\{f^{\prime}\right\}$ which may depend on some model variables. For example, the drag force depends on the element geometry which may change as the element deteriorates to failure ${ }^{34}$.

(c) At the end of the first prediction step the cognate space is identified based on the values of the admixture coefficients ${ }^{33}$. This reduces the modal basis which must be updated at each increment and saves a lot of computational time. Physically, the term cognate space means the space of modes that are related. For example, in the offshore tower shown in Fig. 6 , bending modes constitute a cognate space, those are modes $(1,2)$, $(8,9),(12,13),(15,16)$. Torsional mode space is defined by $(3,18,19)$.

(d) In the correction phase of the algorithm in each increment, correction into the admissible cognate space is performed. At the end of the prediction phase, after computing approximate values of the $a_{e} s$, the force vector $\left.\lambda f^{\prime}\right\}$ and all cognate extracted modes $\lambda\left\{\psi^{\prime}\right\}_{i}$ are updated. Then, the incremental counterparts of eqns (41)-(44) are used to solve for the corrected values of the incremental variables $\iota_{e}, e=1,2, \ldots, p$, subject to upper and lower bounds (59). In future developments, eqns (46)-(49) will be implemented to include buckling and stress global failure equations.

(e) In the incremental approach, attention must be paid to the form of the optimality criterion. If the redundancy norm is reduced incrementally, the optimum of the original problem (eqns (50)-(58)) will be missed. To prevent that, the incremental optimality criterion in increment , should be

$$
\min \sum_{e=1}^{p}\left[\left(1+\alpha_{e}\right) \prod_{q=1}^{1-1}\left(1+{ }_{q} \alpha_{e}\right)-1\right]^{2}
$$

(f) Computer code RESTRUCT is used to implement the algorithm described above and summarized in Fig. 4. RESTRUCT was developed 
initially to solve the problem of structural redesign. It is currently about 27000 Fortran 77 commands. RESTRUCT may serve as postprocessor to any special or general purpose FE code including MSC/NASTRAN.V64 and performs computations with concentrated mass, spring, rod (truss), bar, beam, triangular and quadrilateral plate, and marine riser tubular elements ${ }^{38}$.

\subsection{Failure point identification and redundancy}

Solution to the optimization problems appearing in both phases of the algorithm are achieved by LPSOL, QPSOL, NPSOL or LCSOL ${ }^{40.41}$ for linear, quadratic, nonlinear, or linear constraint optimization problems ${ }^{42}$. The result of course is $S 2_{i}^{*}$ the failure point fully defined for design. This is the advantage of the element geometric redundancy injection achieved by PAR and computed by LEAP. The overall structural redundancy is the value of the optimality criterion. From the geometric injection, any other redundancy injection as defined in Section 3 can be computed.

\section{NUMERICAL APPLICATIONS}

Computations of individual and joint design points for various natural frequency and static deflection criteria are performed by the LEAP algorithm implemented in RESTRUCT for numerous applications on three different structures. The redundancy of the structure is computed in the form of element geometric injections. It is presented, however, only as minimal Euclidean norm for comparison purposes.

\subsection{0-element, 48-dof, clamped-hinged beam}

The 10-element, 48-dof, clamped-hinged beam shown in Fig. 5 is subjected to a concentrated load applied on node 7 in the z-direction, and a uniform load applied in the y-direction. MSC/NASTRAN modal dynamic and static deflection analysis yield $\omega_{1}=183.092 \mathrm{rad} / \mathrm{s}$, horizontal and vertical deflections at node $7 v_{7}=12.151 \mathrm{~mm}$ and $w_{7}=17.733 \mathrm{~mm}$, respectively. The beam properties are shown in Fig. 5 . Model variables are shown in Table 1. Fourteen applications of redundancy analysis are performed and the redundancy results are shown in Table 2. Only the optimal value of the redundancy norm $\|\boldsymbol{\alpha}\|_{2}$ is shown for comparison. It should be obvious by now though the RESTRUCT computes the entire geometric redundancy injection and not just the redundancy. The applications in Table 2 are divided into two 


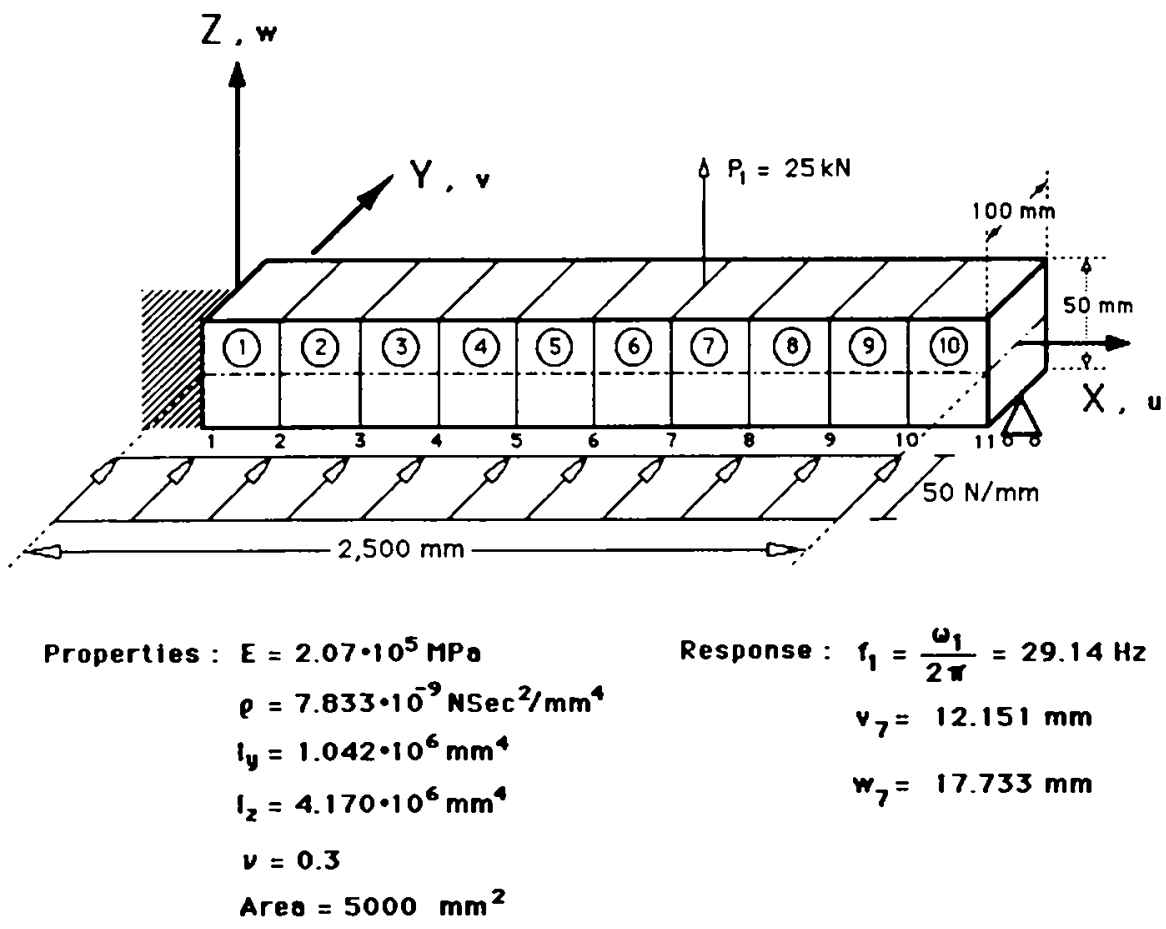

Fig. 5. Clamped-hinged beam: 10 elements, 28 d.o.f.s.

groups. In the first group, quantification of failure criteria is specified as reduction of the first eigenvalue or increase of static deflection by a factor of more than two. Both indicate deterioration of structural stiffness. In the second group of applications, that factor of deterioration is 3.333 . The appropriate general perturbation equations provide the global failure

TABLE 1

Clamped-hinged Beam Model; Structural Groups and Model Variables

\begin{tabular}{|c|c|c|}
\hline $\begin{array}{c}\text { Structural } \\
\text { group } \\
\text { no. }\end{array}$ & $\begin{array}{c}\text { Design variables, } a_{e} \\
p=21\end{array}$ & $\begin{array}{c}\text { Elements } \\
\text { no. }\end{array}$ \\
\hline 1 & $a_{1}\left(a_{l_{y}}\right), a_{2}\left(a_{l_{\gamma}}\right), a_{3}\left(a_{1}\right)$ & 1,2 \\
\hline 2 & $\alpha_{4}\left(\alpha_{l_{y}}\right), \alpha_{5}\left(\alpha_{1}\right), \alpha_{6}\left(\alpha_{A}\right)$ & 3,4 \\
\hline 3 & $\alpha_{7}\left(\alpha_{l_{y}}\right), \alpha_{8}\left(a_{l_{z}}\right), \alpha_{9}\left(\alpha_{A}\right)$ & 5 \\
\hline 4 & $\alpha_{10}\left(a_{I_{y}}\right), a_{11}\left(a_{I_{z}}\right), \alpha_{12}\left(a_{1}\right)$ & 6 \\
\hline 5 & $a_{13}\left(a_{I_{y}}\right), a_{14}\left(a_{l_{z}}\right), a_{15}\left(a_{A}\right)$ & 7 \\
\hline 6 & $a_{16}\left(\alpha_{I_{y}}\right), a_{17}\left(a_{J_{z}}\right), a_{18}\left(\alpha_{A}\right)$ & 8 \\
\hline 7 & $a_{19}\left(a_{l_{y}}\right), \alpha_{20}\left(a_{J_{z}}\right), a_{21}\left(a_{A}\right)$ & 9,10 \\
\hline
\end{tabular}


B. Kang et al.

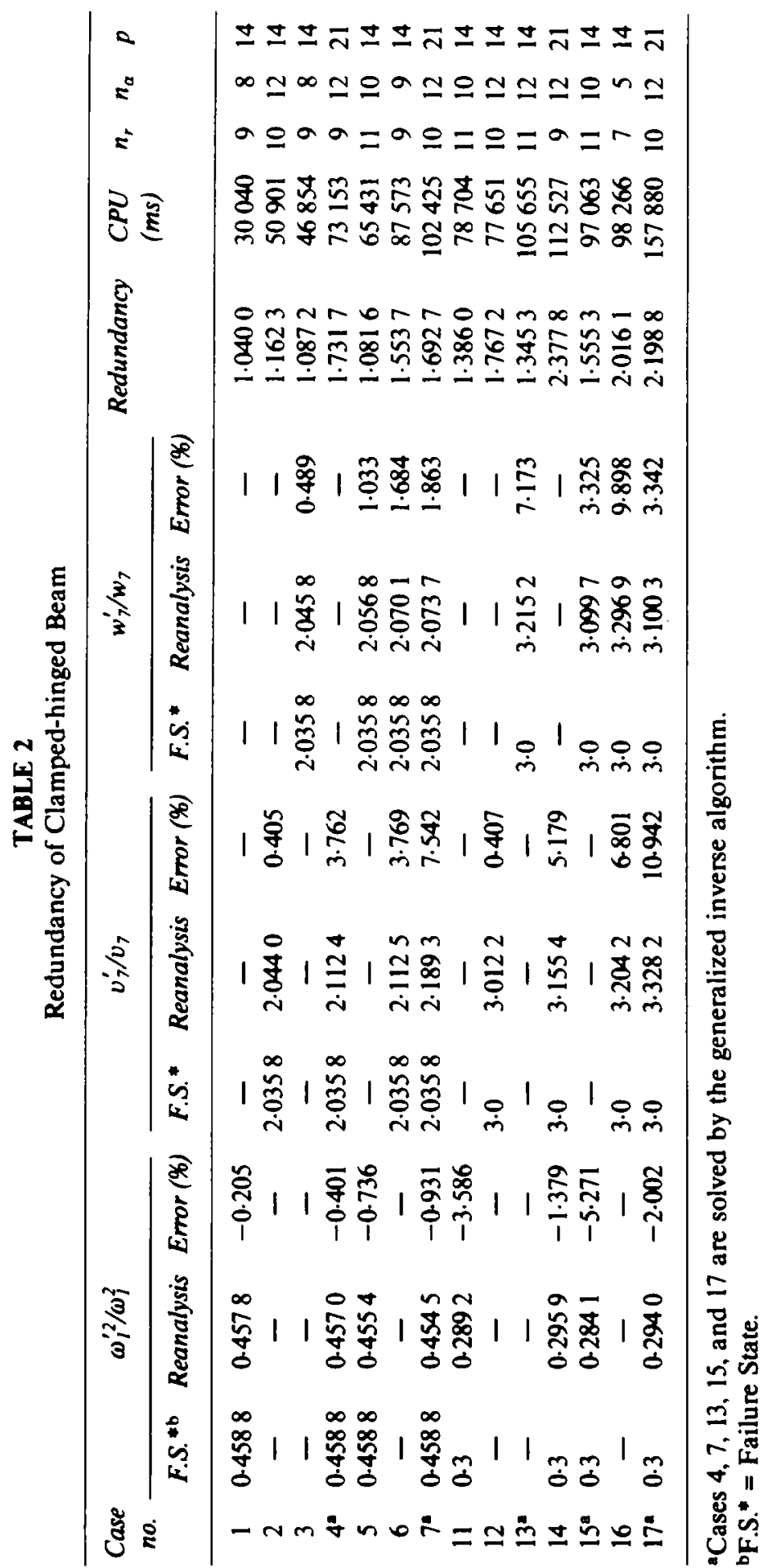


equations and the LEAP algorithm computes the individual or joint failure points in the CPU time shown per case in the table. The University of Michigan UB System (secondary mainframe) IBM 3090 computer was used. The number of extracted modes $n_{r}$ and admissibility conditions $n_{a}$ are also shown.

In all cases, no FE analysis was performed other than that of S1. For each application the failure state (FS), as specified by RESTRUCT in terms of model variables $\alpha$, is compared to reanalysis results obtained by running MSC/NASTRAN.V64. The error column shows the accuracy of the code. In applications 7 and 17, where a triple design point is calculated subject to 12 admissibility conditions and redundancy results are inconsistent between cases 7 and 4 , and 17 and 14 . The error is high actually because there is no solution and a minimum error solution in satisfaction of all constraints is calculated.

\subsection{4-element, 192-dof, offshore tower}

The finite element model of an offshore tower is shown in Fig. 6. The tower is $69.95 \mathrm{~m}$ high above the seabed $(z=0.0)$ and operates in $45.72 \mathrm{~m}$ water depth. The tower at the base is $38.10 \mathrm{~m}$ square and tapers linearly to $22.86 \mathrm{~m}$ at the deck. The FE model of the tower is composed of 104 circular tubular beam elements and has 192 dofs. Loading on the tower is due to: (i) 240 tonnes deck load which is applied to the structure as uniformly distributed load at the deck nodal points; (ii) wave hydrodynamic forces calculated for a design wave of $182.88 \mathrm{~m}$ length and $6.10 \mathrm{~m}$ height using Morison's equation. The wave propagates in the $\mathrm{x}$ direction; (iii) water current generated by wind in the $\mathrm{x}$-direction with linear velocity profile of $1.03 \mathrm{~m} / \mathrm{s}$ at the mean free surface waterline and zero at the sea bed.

Modal dynamic analysis by MSC/NASTRAN has produced $\omega_{1}=$ $\omega_{2}=4.695 \mathrm{rad} / \mathrm{s}$ for bending modes in the $\mathrm{XZ}$ and $\mathrm{YZ}$ planes and $\omega_{3}=5.353 \mathrm{rad} / \mathrm{s}$ for torsional mode with respect to $Z$. Table 3 summarizes information on the tower used in redundancy computations. Six applications were run using RESTRUCT and results are shown in Table 4. Deterioration factors in the first and third eigenvalues of 1.54 and 2 are used to quantify failure. Failure points are again computed by postprocessing FE analysis results for S1 only. Individual and joint design points are computed and redundancy results are shown. The LEAP algorithm in RESTRUCT can be pushed further in this application if errors of more than $3 \%$ are considered acceptable. The alternative for higher accuracy is obvious; $\mathrm{S} 1$ can be redefined closer to 


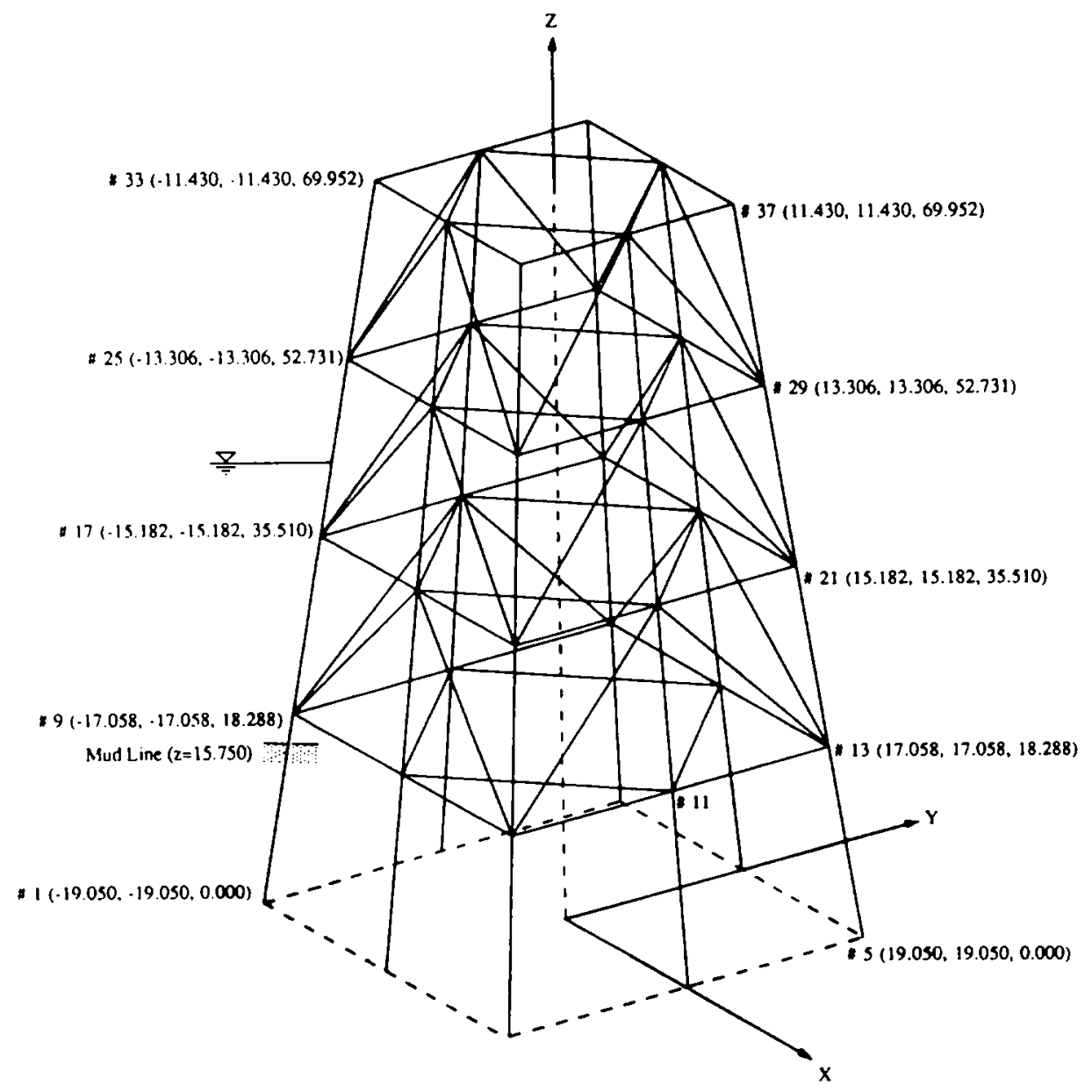

Fig. 6. Offshore tower: 104 elements, 192 d.o.f.s.

the failure point after a few increments. That implies use of one more FE analysis which may not be desirable.

\subsection{5-element, 810 dof, Ambrose tower}

Five structural groups are designated for change in the Ambrose tower ${ }^{43}$ shown in Fig. 7. Those five element sets do not constitute the entire structure and are described in Table 5. Ten model variables $(p=10)$ are used, that is the fractional changes of the moment of inertia and area of each element cross section for each set.

The Ambrose tower is subject to a wind generated water current of 
TABLE 3

Offshore Tower Model; Structural Groups, Model Variables and Dimensions

\begin{tabular}{|c|c|c|c|c|c|}
\hline $\begin{array}{c}\text { Structural } \\
\text { group } \\
\text { no. }\end{array}$ & $\begin{array}{c}\text { Design } \\
\text { variables } \\
a_{e}\end{array}$ & Description & $\begin{array}{c}D_{o} \\
(m)\end{array}$ & $\begin{array}{c}D_{i} \\
(m)\end{array}$ & $\begin{array}{c}\text { Number } \\
\text { of } \\
\text { elements }\end{array}$ \\
\hline 1 & $\begin{array}{l}a_{1}\left(a_{l}\right) \\
a_{2}\left(\alpha_{A}\right)\end{array}$ & $\begin{array}{c}\text { Legs below first } \\
\text { bracing }\end{array}$ & 0.762 & 0.737 & 8 \\
\hline 2 & $\begin{array}{l}a_{3}\left(a_{l}\right) \\
a_{4}\left(a_{A}\right)\end{array}$ & $\begin{array}{l}\text { Legs between first } \\
\text { and second bracing }\end{array}$ & 0.610 & 0.584 & 8 \\
\hline 3 & $\begin{array}{l}a_{5}\left(a_{l}\right) \\
a_{6}\left(a_{A}\right)\end{array}$ & $\begin{array}{l}\text { Legs above second } \\
\text { bracing }\end{array}$ & 0.610 & 0.584 & 16 \\
\hline 4 & $\begin{array}{l}a_{7}\left(a_{1}\right) \\
a_{8}\left(a_{A}\right)\end{array}$ & $\begin{array}{l}\text { Horizontal } \\
\text { bracing }\end{array}$ & 0.483 & 0.464 & 32 \\
\hline 5 & $\begin{array}{c}\alpha_{9}\left(a_{l}\right) \\
\alpha_{10}\left(\alpha_{1}\right)\end{array}$ & $\begin{array}{l}\text { Horizontal cross } \\
\text { bracing }\end{array}$ & 0.508 & 0.489 & 16 \\
\hline 6 & $\begin{array}{l}a_{11}\left(a_{1}\right) \\
a_{12}\left(a_{A}\right)\end{array}$ & $\begin{array}{l}\text { Vertical cross } \\
\text { bracing }\end{array}$ & 0.610 & 0.591 & 24 \\
\hline
\end{tabular}

linear profile with surface velocity $V_{s}=2.5 \mathrm{~m} / \mathrm{s}$ and co-directional monochromatic wave of period $T=10 \mathrm{~s}$ and height $H=3.05 \mathrm{~m}$ propagating in the positive $x$-direction. The modal dynamic and static analyses of S1 were performed by MSC/NASTRAN.V64 and produced $\omega_{1}=5.848 \mathrm{rad} / \mathrm{s}$ and maximum static deflection $\omega_{72}=21.889 \mathrm{~mm}$. Quantification of the first eigenvalue $\omega_{1}^{2}$ and of the maximum static deflection $w_{72}$ provides the two failure criteria. Node 72 is located at the base of the module support frame. The water free surface is located at the lower end of element 8 . Redundancy analysis results for two individual and one joint failure points are shown in Table 6 . The deterioration factors are of the order of 1.28-1.32. Accuracy is very good but it deteriorates slowly for failure points that are further away from S1. In that case, $\mathrm{S} 1$ must be redefined and $\mathrm{FE}$ analysis performed once more.

The accuracy of the algorithm can be improved in many simple ways and many more complex ways. Among the simple ones are: (1) to increase the number of $\alpha_{e} s$ and allow more flexibility to the structure and the optimization algorithm; (2) to reduce the increment size from $7 \%$ to as little as 4\%; (3) to take one more incremental step to match predictions with goals; (4) to redefine state S1 when accuracy is lost because perturbation leaps become too big. A more complex way presented pursued for large scale structures is substructuring. The superelement 
B. Kang et al.

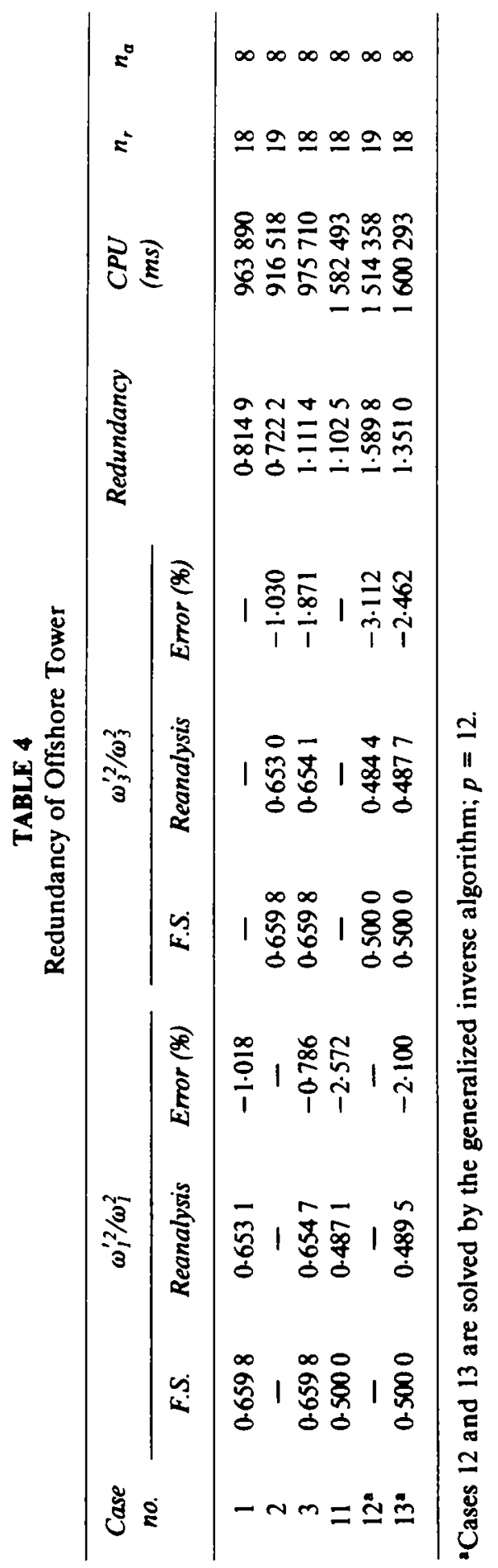




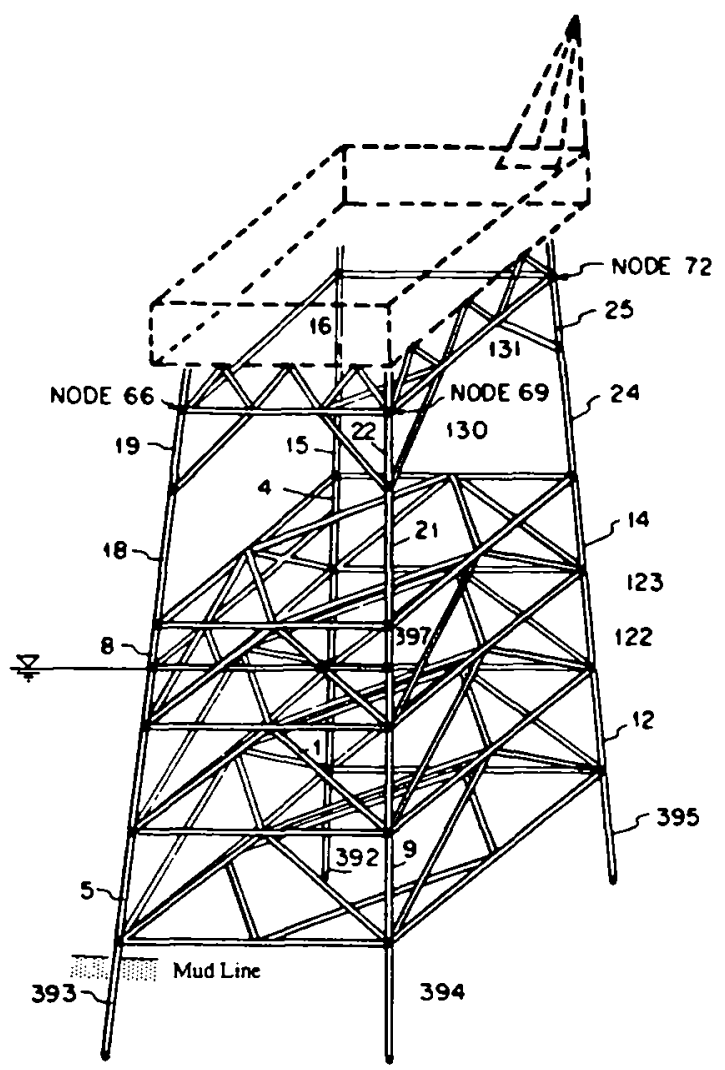

Fig. 7. Ambrose tower: 605 elements, 810 d.o.f.s.

TABLE 5

Ambrose Tower Model; Structural Groups and Model Variables

\begin{tabular}{cccc}
\hline $\begin{array}{c}\text { Structural } \\
\text { group } \\
\text { no. }\end{array}$ & $\begin{array}{c}\text { Design } \\
\text { variables } \\
\alpha_{e}\end{array}$ & Description & $\begin{array}{c}\text { Number } \\
\text { of } \\
\text { elements }\end{array}$ \\
\hline 1 & $\begin{array}{c}a_{1}\left(a_{l}\right) \\
a_{2}\left(a_{A}\right)\end{array}$ & $\begin{array}{c}\text { Legs between joint below } \\
\text { fifth bracing and deck }\end{array}$ & 8 \\
2 & $\begin{array}{c}a_{3}\left(a_{l}\right) \\
a_{4}\left(a_{A}\right)\end{array}$ & $\begin{array}{c}\text { Legs between fourth bracing } \\
\text { and joint below fifth bracing }\end{array}$ & 4 \\
3 & $\begin{array}{c}a_{5}\left(a_{l}\right) \\
a_{6}\left(a_{A}\right)\end{array}$ & $\begin{array}{c}\text { Legs between fourth and third } \\
\text { bracing }\end{array}$ & 7 \\
4 & $\begin{array}{c}a_{7}\left(a_{l}\right) \\
a_{8}\left(a_{A}\right)\end{array}$ & Legs between third and first \\
& $a_{9}\left(a_{l}\right)$ & bracing & 8 \\
5 & $a_{10}\left(a_{A}\right)$ & Legs below first bracing & 4 \\
\hline
\end{tabular}


B. Kang et al.

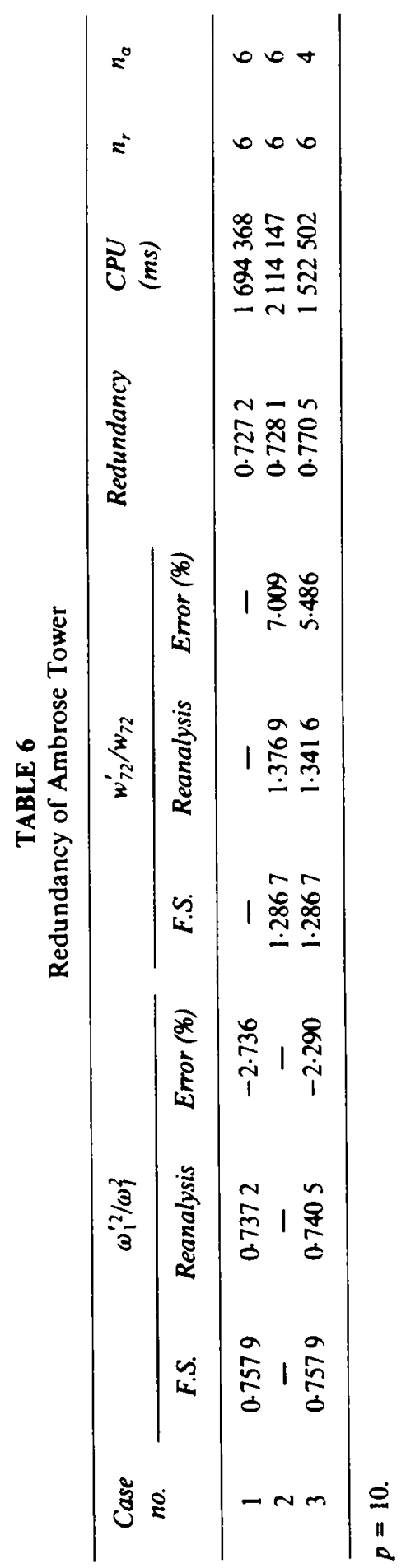


capability of MSC/NASTRAN.V66 allows for substructuring. A supercomputer version of RESTRUCT runs presently on the San Diego supercomputer facility and postprocesses MSC/NASTRAN.V66 data.

\section{CONCLUSIONS AND IMPACT ON DESIGN}

A methodology has been presented for computation of structural redundancy of deterministic structures. The methodology is called PAR (Perturbation Approach to Redundancy) because perturbation is used to relate two structural states, the initial (intact/damaged) and the failure point. PAR produces global failure equations and brings the level of sophistication of structural analysis in redundancy computations up to par with the FE method used in design practice. Thus, the introduced methodology is valid for any structure, small or large, discrete or continuous, that can be analyzed by the FE method. LEAP (LargE Admissible Perturbations) theory is used in this methodology to relate the two structural states involved, solve the global failure equations, and compute the failure point by postprocessing data of the only FE a nalysis performed, that of the initial structure.

The concept of invariant and consistent redundancy has been introduced by defining redundancy injections, mappings, and measures for the FE model variables, structural properties, or performance. In the LEAP algorithm developed, the connectivity of elements is preserved in the FE model and admissibility conditions are forced on the modal basis, thus ensuring that the final state specified (failure point) is a real structure. Invariance and consistency of redundancy, element connectivity, and perturbation admissibility set the basis for relating redundancy in performance to structural and geometric redundancy injectively, and therefore appropriately for design.

The numerical applications in this paper have shown the present status of LEAP theory and its ability to support the PAR methodology introduced in this work. Even though the LEAP theory has been developed for more than 7 years and has evolved from the linear FE structural perturbation theory introduced in 1975, it is an open ended research area like the FE method. There is a plethora of two-state problems that can be solved by LEAP. On the other hand, this is the first paper on PAR and there are several issues important in structural redundancy and reliability that have not been implemented in the computer code RESTRUCT. Element failure detection and model reconfiguration, stress, and global buckling failure are three of those issues. It was explained, however, that the PAR methodology can resolve 
those issues and meet the present research challenges in redundancy analysis and design of large scale structures. Thus, the large admissible perturbation approach to redundancy presented in the paper introduces a powerful alternative to the two major methods for structural reliability analysis, that is the systems approach and the stochastic FE method.

For improving LEAP theory to solve two-state problems, we pursue presently the following research goals. (1) Improve the computational efficiency of RESTRUCT to reduce the CPU time. Tables $2,4,6$ show that the CPU time is high even though it is small compared with trial and error with repeated FE analyses. We have spotted six aspects of computational inefficiency in RESTRUCT and we expect to be able to reduce CPU by a factor of $2 \cdot 5-3 \cdot 5$. The most important development from the computational point of view is to identify elements of small static strain and modal dynamic energy content and exclude those from the LEAP-PAR process: We have not established as yet a solid theoretical basis for this idea. Our computations, however, based on the offshore tower in Fig. 6, show a CPU time reduction by a factor of 1.5 with trivial loss in accuracy when we exclude from the process many small elements with total energy content of $10 \%$. (2) Implement substructuring in order to analyze larger scale structures with RESTRUCT. (3) Use energy concepts in RESTRUCT to predict loss of accuracy of the modal basis as the final structural state moves away from the initial state. This will automate the process of redefining Sl and performing one intermediate FE analysis if necessary. Note that in the numerical applications in this paper only one FE analysis was performed; there was no need to redefine State $\mathrm{S} 1$.

For improving the PAR methodology we pursue presently the following goals. (4) Develop an analogous methodology for reliability analysis of large scale continuous structures. (5) Implement stress failure criteria (eqn 46), and element failure detection and model reconfiguration in RESTRUCT. (6) Implement structural collapse as a failure criterion $\left(\omega_{i}^{\prime}=0\right.$ in eqn 41). (7) Develop a global buckling failure LEAP algorithm using a buckling modal expansion, (eqns $47-49$ ) rather than a modal dynamics basis.

\section{ACKNOWLEDGMENTS}

The work on the Large Admissible Perturbation (LEAP) theory is supported by the Office of Naval Research through Grant No. DOD-GN00014-90-J-4081 and is part of the first author's Ph.D. dissertation. The work on the Perturbation Approach to Redundancy (PAR) is part of the 
second author's Ph.D. dissertation and is supported by The University of Michigan/Sea Grant/Industry Consortium in Offshore Engineering under Michigan Sea Grant College Program, projects number E/GLE14 and R/T-23, under Grant Number NA85AA-D-SG045C from the Office of Sea Grant, National Oceanic and Atmospheric Administration (NOAA), U.S. Department of Commerce, and funds from the State of Michigan. Industry participants include the American Bureau of Shipping; ARCO Gas and Oil Company; Conoco, Inc.; Exxon Production Research; Mobil Research and Development; Shell Companies Foundation; and the U.S. Coast Guard. The U.S. Government is authorized to produce and distribute reprints for governmental purposes notwithstanding any copyright notation appearing hereon.

Challenging questions and constructive suggestions from Professors W. S. Vorus and M. G. Parsons of the Department of Naval Architecture and Marine Engineering of the University of Michigan have contributed greatly to development of the Perturbation Approach to Redundancy. Discussions with Dr. D. Karsan of Conoco Inc., Dr. Y. N. Chen of the Research and Development Division of ABS, and Professor E. Nikolaidis of the Department of Aerospace and Ocean Engineering of Virginia Polytechnic Institute and State University have provided valuable guidance.

\section{REFERENCES}

1. Lloyd, J. R. \& Clawson, W. C., Reserve and residual strength of pile-founded offshore platforms, Proceedings of Design-Inspection-Redundancy Symposium, Williamsburg, Virginia, November 1983, pp. 8.1-8.37.

2. D'Oliveira, J. G. \& Zimmer, R. A., Redundancy considerations in the structural design of floating offshore platforms, Proceedings of DesignInspection-Redundancy Symposium, Willia msburg, Virginia, November 1983, pp. 13.1-13.31.

3. Nikolaidis, E. \& Kapania, R. K., System reliability and redundancy of marine structures: A review of the state of the art, Journal of Ship Research, 34(1) (March 1990) pp. 48-59.

4. Stiansen, S. G., Interrelation between design, inspection and redundancy in marine structures, Proceedings of Design-Inspection-Redundancy Symposium. Williamsburg, Virginia, November 1983, pp. 2.1-2.22.

5. Frangopol, D. M. \& Curley, J. P., Effects of damage and redundancy on structural reliability, Journal of Structural Engineering, ASCE, 113(7) (1987) pp. 1533-49.

6. Fu, G. \& Moses, F., Probabilistic concepts of redundancy and damage tolerability for structural systems, Proceedings, Sth International Conference on 
Structural Safety and Reliability, ICOSSAR '89, Aug. 1989, San Francisco, pp. 967-75.

7. Hughes, O., Ship Structural Design, Wiley-Interscience, 1983.

8. De, R. S., Offshore Structural System Reliability: Wave-Load Modeling, System Behavior, and Analysis, Report No. RMS-6, Dept. of Civil Engineering, Stanford University, March 1990.

9. De, R.S., Karamchandani, A. \& Cornell, C. A., Study of redundancy in nearideal parallel structural systems, Proceedings, 5th International Conference on Structural Safety and Reliability, ICOSSAR '89, Aug. 1989, San Francisco, pp. 975-83.

10. Nordal, H., Cornell, C. A. \& Karamchandani, A., A structural system reliability case study of an eight-leg steel jacket offshore production platform, Proceedings of Marine Structural Reliability Symposium. SNAME, Arlington, Virginia, October 5-6, 1987, pp. 193-216.

11. Paliou, C., Shinozuka, M. \& Chen, Y.-N., Reliability analysis of offshore structures, Proceedings of Marine Structural Reliability Symposium, SSC and SNAME, Arlington, VA, October 5-6, 1987.

12. Bennett, R. M., Ang, A. H.-S. \& Goodpasture, D. W., Probability safety assessment of redundant bridges, Proceedings of ICOSSAR '85, Kobe, Japan.

13. Feng, Y. S. \& Moses, F., Optimum design, redundancy and reliability of structural systems, Computers and Structures, 24(2) (1986) pp. 239-51.

14. Thoft-Christensen, P. \& Murotsu, Y., Application of Structural Systems Reliability Theory, Springer-Verlag, Berlin, 1986.

15. Smith, C. S., Structural redundancy and damage tolerance in relation to ultimate ship-hull strength, Proceedings of Design-Inspection-Redundancy Symposium, Williamsburg, Virginia, November 1983, pp. 5.1-5.22.

16. Marshall, P. W., The design-inspection-redundancy triangle, Proceedings of Design-Inspection-Redundncy Symposium, Williamsburg, Virginia, November 1983, pp. 1.1-1.8.

17. Mikesell, W. R., Pieper, C. J. \& Whipple, R. A., Design, inspection and redundancy of pressure vessels, Proceedings of Design-Inspection-Redundancy Symposium, Williamsburg, Virginia, November 1983, pp. 16.1-16.22.

18. Jubb, J. E., Strategies for assessing design and inspection requirements for redundant structures, Proceedings of Design-Inspection-Redundancy Symposium, Williamsburg, Virginia, November 1983, pp. 6.1-6.7.

19. Weidler, J. B., Unified strategy of design, redundancy and inspection of fixed offshore platforms, Proceedings of Design-Inspection-Redundancy Symposium, Williamsburg, Virginia, November, 1983, pp. 10.1-10.27.

20. Pittalunga, A., Reliability based ship design in the 90 's; releastic scenario or dream?, Proceedings of International Symposium on Advanced Research for Ships and Shipping in the Nineties, S. Margherita Ligure, Genova, October 1-3, 1987.

21. Mansour, A. E., Jan, H. Y., Zigelman, C. I., Chen, Y. N. \& Harding, S. J., Implementation of reliability methods to marine structures, SNAMETrans, 92 (1984) pp. 353-82.

22. Stetson, K A., Perturbation method of structural design relevant to holographic vibration analysis, ALAA Journal, 13(4) (April 1975), pp. 457-9.

23. Stetson, K. A., Harrison, I. R. \& Palma, G. E., Redesigning structural vibration modes by inverse perturbation subject to minimal change theory, 
Computer Methods in Applied Mechanics and Engineering, 16(2) (November 1978) pp. 151-75.

24. Stetson, K. A. \& Harrison, I. R., Redesign of structural vibration modes by finite-element inverse perturbation, ASME Transactions, Journal of Engineering for Power, 103(2) (April 1981) pp. 319-25.

25. Kim, J. H. \& Bernitsas, M. M., Redesign of marine structures by perturbation, Journal of Marine Structures, 1(2)(September 1988) pp. 139-83.

26. Bernitsas, M. M. \& Kang, B. S., Admissible large perturbations in structural redesign, ALAA Journal, 29(1) (January 1991) pp. 104-113.

27. Stetson, K. A. \& Palma, G. E., Inversion of first-order perturbation theory and its application to structural design, ALAA Joumal, 14(4) (April 1976) pp. 454-460.

28. Sandström, R. E. \& Anderson, W. J., Modal perturbation methods for marine structures, SNAME Trans, 90 (1982) pp. 41-54.

29. Haug, E. J., Choi, K. K. \& Komkov, V., Design Sensitivity Analysis of Structural Systems, Academic Press, 1986.

30. Hoff, C. J., Bernitsas, M. M., Sandström, R. E. \& Anderson, W. J., Nonlinear incremental inverse perturbation method for structural redesign, Proceedings of ALAA/ASME/ASCE/AHS 24th Structures, Structural Dynamics and Materials Conference, Lake Tahoe, Nevada, May 1983; ALAA Joumal, 22(9) (September 1984) pp. 1304-9.

31. Bernitsas, M. M., Hoff, C. J. \& Kokarakis, J. E., Nonlinear inverse perturbation in structural redesign of risers, Proceedings of the 3rd Offshore Mechanics and Arctic Engineering Symposium, New Orleans, Louisiana, February 12-14, 1984; also ASME Transactions, Journal of Energy Resources Technology, 107(2) (June 1985) pp. 256-63.

32. Hoff, C. J. \& Bernitsas, M. M., Dynamic redesign of marine structures, Journal of Ship Research, 29(4) (December 1985) pp. 285-295.

33. Bernitsas, M. M. \& Tawekal, R. L., Structural model correlation using large admissible perturbations in cognate space, ALAA Journal, 29(6)(June 1991).

34. Tawekal, R. L. \& Bernitsas, M. M., Finite element model correlation for offshore structures, Proceedings 10th International OMAE Conference, Norway, June 1991.

35. Gans, H. D. \& Anderson, W. J., Structural optimization incorporating centrifugal and coriolis effects, Proceedings of 30th ALAA/ASME/ASCE/AHS/ ASC Structures, Structural Dynamics and Materials Conference, Mobile Alabama, 3-5 April 1989, pp. 1311-20.

36. Vanmarcke, E., Shinozuka, M., Nakagiri, S., Schueller, G. I. \& Grigoriu, M., Random fields and stochastic finite elements, Structural Safety, 3 (1986) pp. 143-166.

37. Liu, P.-L. \& Der Kiureghian, A., Finite element reliability of two dimensional continua with geometrical nonlinearity, Proceedings, 5th International Conference on Structural Safety and Reliability, ICOSSAR '89, Aug. 1989, San Francisco, pp. 1089-96.

38. Bernitsas, M. M., Kang, B. S. \& Tawekal, R., RESTRUCT Version 3.0: A Program for Redesign of Structures, Report to the University of Michigan/Sea Grant/Industry Consortium in Offshore Engineering, and Publication No. 312, Department of Naval Architecture and Marine Engineering, the University of Michigan, Ann Arbor, October 1989. 
39. Kreyszig, E., Introductory Functional Analysis with Applications, Wiley, 1978.

40. Gill, P. E., Murray, W., Saunders, M. A. \& Wright, M. H., User's Guide for SOL/QPSOL: A Fortran Package for Quadratic Programming, Department of Operations Research, Stanford University, 1983.

41. Gill, P. E., Murray, W., Saunders, M. A. \& Wright, M. H., User's Guide for SOL/NPSOL: A Fortran Package for Nonlinear Programming, Department of Operations Research, Stanford University, 1983.

42. Luenberger, D. G., Introduction to Linear and Nonlinear Programming, Addison-Wesley, 1973.

43. Wojnarowski, M. E., Stiansen, S. G. \& Reddy, N. E., Structural Integrity Evaluation of a Fixed Platform Using Vibration Criteria, Paper No. OTC2909, Ninth Annual Offshore Technology Conference, Houston, May 1977, Vol. III, pp. 247-56.

44. Madsen, H. O., Krenk, S. \& Lind, N. C., Methods of Structural Safety, PrenticeHall, Englewood Cliffs, NJ, 1986. 